\title{
MINIMAL ZERO-SUM SEQUENCES OF LENGTH FOUR OVER FINITE CYCLIC GROUPS II
}

\author{
YUANLIN LI* AND JIANGTAO PENG
}

\begin{abstract}
Let $G$ be a finite cyclic group. Every sequence $S$ over $G$ can be written in the form $S=\left(n_{1} g\right) \cdot \ldots \cdot\left(n_{l} g\right)$ where $g \in G$ and $n_{1}, \ldots, n_{l} \in[1, \operatorname{ord}(g)]$, and the index ind $(S)$ of $S$ is defined to be the minimum of $\left(n_{1}+\cdots+n_{l}\right) / \operatorname{ord}(g)$ over all possible $g \in G$ such that $\langle g\rangle=G$. An open problem on the index of length four sequences asks whether or not every minimal zero-sum sequence of length 4 over a finite cyclic group $G$ with $\operatorname{gcd}(|G|, 6)=1$ has index 1. In this paper, we show that if $G=\langle g\rangle$ is a cyclic group with order of a product of two prime powers and $\operatorname{gcd}(|G|, 6)=1$, then every minimal zero-sum sequence $S$ of the form $S=(g)\left(n_{2} g\right)\left(n_{3} g\right)\left(n_{4} g\right)$ has index 1. In particular, our result confirms that the above problem has an affirmative answer when the order of $G$ is a product of two different prime numbers or a prime power, extending a recent result by the first author, Plyley, Yuan and Zeng.
\end{abstract}

\section{INTRODUCTION}

Throughout this paper, let $G$ be an additively written finite cyclic group of order $|G|=n$. By a sequence over $G$ we mean a finite sequence of terms from $G$ which is unordered and repetition of terms is allowed. We view sequences over $G$ as elements of the free abelian moniod $\mathcal{F}(G)$ and use multiplication notation. Thus a sequence $S$ of length $|S|=k$ is written in the form $S=\left(n_{1} g\right) \cdot \ldots \cdot\left(n_{k} g\right)$ where $n_{1}, \ldots, n_{k} \in \mathbb{N}$ and $g \in G$. We call $S$ a zero-sum sequence if the sum of $S$ is zero (i.e. $\sum_{i=1}^{k} n_{i} g=0$ ). If $S$ is a zero-sum sequence, but no proper nontrivial subsequence of $S$ has sum zero, then $S$ is called a minimal zero-sum sequence. Recall that the index of a sequence $S$ over $G$ is defined as follows.

Definition 1.1. For a sequence over $G$

$$
S=\left(n_{1} g\right) \cdot \ldots \cdot\left(n_{l} g\right), \text { where } 1 \leq n_{1}, \ldots, n_{l} \leq n,
$$

the index of $S$ is defined by $\operatorname{ind}(S)=\min \left\{\|S\|_{g} \mid g \in G\right.$ with $\left.G=\langle g\rangle\right\}$ where

$$
\|S\|_{g}=\frac{n_{1}+\cdots+n_{l}}{\operatorname{ord}(g)} \text {. }
$$

2000 Mathematics Subject Classification. Primary 11B30 11B50 20K01.

Key words and phrases: minimal zero-sum sequences, index of sequences.

This research was supported in part by a Discovery Grant from the Natural Sciences and Engineering Research Council of Canada, the National Natural Science Foundation of China (No. 11126137 and No. 11271250) and a research grant from Civil Aviation University of China.

*Corresponding author: Yuanlin Li, Department of Mathematics, Brock University, St. Catharines, Ontario Canada L2S 3A1, Fax:(905) 378-5713;

E-mail: yli@brocku.ca (Y. Li)

October 17, 2018. 
Clearly, $S$ has sum zero if and only if $\operatorname{ind}(S)$ is an integer. There are also slightly different definitions of the index in the literature, but they are all equivalent (see Lemma 5.1.2 in [7]).

The index of a sequence is a crucial invariant in the investigation of (minimal) zero-sum sequences (resp. of zero-sum free sequences) over cyclic groups. It was first addressed by Kleitman-Lemke (in the conjecture [9, page 344]), used as a key tool by Geroldinger ([6, page $736]$ ), and then investigated by Gao [3] in a systematical way. Since then it has received a great deal of attention (see for example [1, 2, 4, 7, 10, 11, 12, 13, 14]).

A main focus of the investigation of index is to determine minimal zero-sum sequences of index 1. If $S$ is a minimal zero-sum sequence of length $|S|$ such that $|S| \leq 3$ or $|S| \geq\left\lfloor\frac{n}{2}\right\rfloor+2$, then $\operatorname{ind}(\mathrm{S})=1$ (see [1, 12, 14]). In contrast to that, it was shown that for each $k$ with $5 \leq k \leq\left\lfloor\frac{n}{2}\right\rfloor+1$, there is a minimal zero-sum sequence $T$ of length $|T|=k$ with $\operatorname{ind}(T) \geq 2$ ([12, 14]) and that the same is true for $k=4$ and $\operatorname{gcd}(n, 6) \neq 1$ ([1] $)$. The only unsolved case is that whether or not every minimal zero-sum sequence of length 4 in a cyclic group $G$ with $\operatorname{gcd}(|G|, 6)=1$ has index 1 and this leads to the following open problem.

Problem 1.2. Let $G$ be a finite cyclic group such that $\operatorname{gcd}(|G|, 6)=1$. Is it true that every minimal zero-sum sequence $S$ over $G$ of length $|S|=4$ has $\operatorname{ind}(S)=1$ ?

In a recent paper [10 the first author together with Plyley, Yuan, and Zeng proved that the open problem (Problem 1.2) has an affirmative answer if $n$ is a prime power. However, the general case is still open. In this paper, we attempt to answer this problem affirmatively for a more general case when $n$ is a product of two prime powers. Our main result is as follows.

Theorem 1.3. Let $n=p_{1}^{\alpha} \cdot p_{2}^{\beta}$, where $p_{1} \neq p_{2}$ are primes and $\alpha, \beta \in \mathbb{N}$, and $\operatorname{gcd}(n, 6)=1$. Let $S=(g)\left(x_{2} g\right)\left(x_{3} g\right)\left(x_{4} g\right)$ be a minimal zero-sum sequence over $G$ such that $\operatorname{ord}(g)=n$ and $1 \leq x_{2}, x_{3}, x_{4} \leq n-1$. Then $\operatorname{ind}(S)=1$.

As applications of Theorem 1.3, we obtain that the problem has an affirmative answer for the group with order of a product of two primes (Theorem 4.4) as well as for the group of prime power order (Theorem 4.2 the main result of [10]).

The paper is organized as follows. In next section, we provide some preliminary results, and then a proof of our main result is given in Section 3. In the last section, we give some applications of Theorem 1.3 .

\section{Preliminaries}

We first list some useful facts and simple results in the following remark. We denote by $|x|_{n}$ the least positive residue of $x$ modulo $n$, where $n \in \mathbb{N}$ and $x \in \mathbb{Z}$.

Remark 2.1. Let $S=\left(x_{1} g\right)\left(x_{2} g\right)\left(x_{3} g\right)\left(x_{4} g\right)$ be a minimal zero-sum sequence over $G$ such that $\operatorname{ord}(g)=n=|G|$, and $1 \leq x_{1} \leq x_{2} \leq x_{3} \leq x_{4} \leq n-1$. Then $x_{1}+x_{2}+x_{3}+x_{4}=\nu n$, where $1 \leq \nu \leq 3$. 
(0). To show that ind $(S)=1$, it suffices to find an integer $m$ with $\operatorname{gcd}(m, n)=1$ such that $\left|m x_{1}\right|_{n}+\left|m x_{2}\right|_{n}+\left|m x_{3}\right|_{n}+\left|m x_{4}\right|_{n}=n$, and this fact will be frequently used later. Furthermore, we may always assume that $\nu \geq 2$.

(1). It was mentioned in [11 that Problem 1.2 was confirmed computationally to hold true if $n \leq 1000$ (The claim has been double checked by the second author and Wang by using a computer program). Hence, throughout the paper, we always assume that $n>1000$.

(2). If $x_{1}+x_{2}+x_{3}+x_{4}=3 n$, then $\left|(n-1) x_{1}\right|_{n}+\left|(n-1) x_{2}\right|_{n}+\left|(n-1) x_{3}\right|_{n}+\left|(n-1) x_{4}\right|_{n}=$ $\left(n-x_{1}\right)+\left(n-x_{2}\right)+\left(n-x_{3}\right)+\left(n-x_{4}\right)=n$. Since $\operatorname{gcd}(n, n-1)=1$, we have ind $(S)=1$. Thus we may always assume that $x_{1}+x_{2}+x_{3}+x_{4}=2 n$.

(3). If $x_{1} \leq x_{2} \leq x_{3}<\frac{n}{2}$, then $x_{4}=2 n-\left(x_{1}+x_{2}+x_{3}\right)>\frac{n}{2}$. Now $\left|(n-2) x_{1}\right|_{n}+\left|(n-2) x_{1}\right|_{n}+$ $\left|(n-2) x_{1}\right|_{n}+\left|(n-2) x_{1}\right|_{n}=\left(n-2 x_{1}\right)+\left(n-2 x_{2}\right)+\left(n-2 x_{3}\right)+\left(2 n-2 x_{4}\right)=n$. Since $\operatorname{gcd}(n, n-2)=1$, we have $\operatorname{ind}(S)=1$.

(4). If $x_{4} \geq x_{3} \geq x_{2}>\frac{n}{2}$, then $x_{1}<\frac{n}{2}$. Since $\left|2 x_{1}\right|_{n}+\left|2 x_{1}\right|_{n}+\left|2 x_{1}\right|_{n}+\left|2 x_{1}\right|_{n}=2 x_{1}+\left(2 x_{2}-\right.$ $n)+\left(2 x_{3}-n\right)+\left(2 x_{4}-n\right)=n$ and $\operatorname{gcd}(n, 2)=1$, we have $\operatorname{ind}(S)=1$.

Let $S$ be the sequence as described in Theorem 1.3, By the above remark, we may always assume that $1+x_{2}+x_{3}+x_{4}=2 n$ and $1<x_{2}<n / 2<x_{3} \leq x_{4}<n-1$. Now let $c=x_{2}, b=$ $n-x_{3}, a=n-x_{4}$, and it is not hard to show that the following proposition implies Theorem 1.3 .

Proposition 2.2. Let $n=p_{1}^{\alpha} \cdot p_{2}^{\beta}$, where $p_{1} \neq p_{2}$ are primes and $\alpha, \beta \in \mathbb{N}$, and $\operatorname{gcd}(n, 6)=1$. Let $S=(g)(c g)((n-b) g)((n-a) g)$ be a minimal zero-sum sequence over $G$ with $\operatorname{ord}(g)=n$, $1+c=a+b$ and $1<a \leq b<c<\frac{n}{2}$. Then $\operatorname{ind}(S)=1$.

For any real numbers $a<b \in \mathbb{R}$, we set $[a, b]=\{x \in \mathbb{Z} \mid a \leq x \leq b\}$ the set of all integers between $a$ and $b$, and similarly, set $[a, b)=\{x \in \mathbb{Z} \mid a \leq x<b\}$. From now (until the end of the next section) we always assume that $S$ is the sequence as described in Proposition 2.2. Next we give a crucial lemma.

Lemma 2.3. Proposition 2.2 holds if one of the following conditions holds:

(1). There exist positive integers $k, m$ such that $\frac{k n}{c} \leq m \leq \frac{k n}{b}, \operatorname{gcd}(m, n)=1,1 \leq k \leq b$, and $m a<n$.

(2). There exists a positive integer $M \in\left[1, \frac{n}{2}\right]$ such that $\operatorname{gcd}(M, n)=1$ and at least two of the following inequalities hold:

$|M a|_{n}>\frac{n}{2},|M b|_{n}>\frac{n}{2},|M c|_{n}<\frac{n}{2}$.

Proof. (1). If $\operatorname{gcd}(m, n)=1$ and $1 \leq k \leq b$, we conclude that $\frac{k n}{c}<m<\frac{k n}{b}$. Since $|m|_{n}+|m c|_{n}+$ $|m(n-b)|_{n}+|m(n-a)|_{n} \leq m+(m c-k n)+(k n-m b)+(n-m a)=n$, we have ind $(S)=1$.

(2). It follows that at least three elements of $\left\{|M|_{n},|M c|_{n},|M(n-b)|_{n},|M(n-a)|_{n}\right\}$ are less than $\frac{n}{2}$. By Remark 2.1 (3), we have ind $(S)=1$.

As a consequence of Lemma 2.3, we have the following easy observation.

Lemma 2.4. If there exist integers $k$ and $m$ such that $\frac{k n}{c} \leq m \leq \frac{k n}{b}, \operatorname{gcd}(m, n)=1$ and $a \leq \frac{b}{k}$, then Proposition 2.2 holds.

Proof. Note that $m<\frac{k n}{b}$. Since $m a<\frac{k n}{b} \times \frac{b}{k}=n$, the result follows from Lemma 2.3 (1). 
In what follows, we assume that $s=\left\lfloor\frac{b}{a}\right\rfloor$. Then we have $s \leq \frac{b}{a}<s+1$ and

$$
\frac{n}{2 a}<\frac{(s+1) n}{2 b}<\cdots<\frac{(2 s-1) n}{2 b}<\frac{s n}{b} \leq \frac{n}{a} .
$$

Since $b<\frac{n}{2}$, we have $\frac{n}{2 b}=\frac{(2 s-t) n}{2 b}-\frac{(2 s-t-1) n}{2 b}>1$, and then $\left[\frac{(2 s-t-1) n}{2 b}, \frac{(2 s-t) n}{2 b}\right]$ contains at least one integer for every $t \in[0, s-1]$.

Now we are ready to give two sufficient conditions for Proposition 2.2 to hold. The first is " $s \geq 8$ " (which follows from Lemmas 2.5 and 2.6) and the other is " $a=2$ " (Lemma 2.7).

Lemma 2.5. Suppose $s \geq 2$ and $\left[\frac{(2 s-2 t-1) n}{2 b}, \frac{(s-t) n}{b}\right]$ contains an integer co-prime to $n$ for some $t \in\left[0,\left\lfloor\frac{s}{2}\right\rfloor-1\right]$. Then Proposition [2.2 holds.

Proof. Suppose $M \in\left[\frac{(2 s-2 t-1) n}{2 b}, \frac{(s-t) n}{b}\right]$ for some $t \in\left[0,\left\lfloor\frac{s}{2}\right\rfloor-1\right]$ and $\operatorname{gcd}(M, n)=1$. Then $M<\frac{s n}{b} \leq \frac{n}{a} \leq \frac{n}{2}$. Note that $\frac{n}{2 a}<\frac{(2 s-2 t-1) n}{2 b}<M<\frac{(s-t) n}{b} \leq \frac{n}{a}$. Hence $|M a|_{n}>\frac{n}{2}$. Also, since $\frac{(2 s-2 t-1) n}{2 b}<M<\frac{(s-t) n}{b}$, we have $\frac{(2 s-2 t-1) n}{2}<M b<(s-t) n$, so $|M b|_{n}>\frac{n}{2}$. It follows from Lemma 2.3 (2) that Proposition 2.2 holds.

Lemma 2.6. Suppose $s \geq 2$ and $\left[\frac{(2 s-2 t-1) n}{2 b}, \frac{(s-t) n}{b}\right]$ contains no integers co-prime to $n$ for every $t \in\left[0,\left\lfloor\frac{s}{2}\right\rfloor-1\right]$. Then the following results hold.

(i). $\frac{n}{2 b}<3$ (where $\frac{n}{2 b}$ is the length of the interval $\left[\frac{\left(2 s-t_{1}-1\right) n}{2 b}, \frac{\left(2 s-t_{1}\right) n}{2 b}\right]$ for each $t_{1} \in[0, s-1]$ ).

(ii). If $s \geq 4$, then $\left[\frac{(2 s-2 t-1) n}{2 b}, \frac{(s-t) n}{b}\right]$ contains exactly one integer for every $t \in\left[0,\left\lfloor\frac{s}{2}\right\rfloor-1\right]$. Furthermore, $\frac{n}{2 b}<2$.

(iii). Suppose that $s \geq 4, x \in\left[\frac{(2 s-2 t-1) n}{2 b}, \frac{(s-t) n}{b}\right]$ and $y \in\left[\frac{(2 s-2 t-3) n}{2 b}, \frac{(s-t-1) n}{b}\right]$ for some $t \in$ $\left[0,\left\lfloor\frac{s}{2}\right\rfloor-2\right]$. Then $\operatorname{gcd}(x, y, n)=1$.

(iv). Suppose that $s \geq 6, x \in\left[\frac{(2 s-2 t-1) n}{2 b}, \frac{(s-t) n}{b}\right]$ and $z \in\left[\frac{(2 s-2 t-5) n}{2 b}, \frac{(s-t-2) n}{b}\right]$ for some $t \in$ $\left[0,\left\lfloor\frac{s}{2}\right\rfloor-3\right]$. Then $\operatorname{gcd}(x, z, n)>1$ and $5 \mid \operatorname{gcd}(x, z, n)$. Furthermore, $z=x-5$ and $\frac{n}{2 b}<\frac{7}{5}$. (v). $s \leq 7$.

Proof. (i). Since $\left[\frac{(2 s-2 t-1) n}{2 b}, \frac{(s-t) n}{b}\right]$ contains no integers co-prime to $n$ for every $t \in\left[0,\left\lfloor\frac{s}{2}\right\rfloor-1\right]$ and $n=p_{1}^{\alpha} \cdot p_{2}^{\beta}$, we have that $\left[\frac{(2 s-2 t-1) n}{2 b}, \frac{(s-t) n}{b}\right]$ contains at most two integers and hence $\frac{n}{2 b}=\frac{(s-t) n}{b}-\frac{(2 s-2 t-1) n}{2 b}<3$.

Next, we assume that $s \geq 4$, so $\left\lfloor\frac{s}{2}\right\rfloor-1 \geq 1$.

(ii). Assume to the contrary that $\left[\frac{(2 s-2 t-1) n}{2 b}, \frac{(s-t) n}{b}\right]$ contains two integers, say $x, x+1$, for some $t \in\left[0,\left\lfloor\frac{s}{2}\right\rfloor-1\right]$. Then $\operatorname{gcd}(x, n)>1$ and $\operatorname{gcd}(x+1, n)>1$. Since $n=p_{1}^{\alpha} \cdot p_{2}^{\beta}$ and $\operatorname{gcd}(n, 6)=1$, for every $z \in[x-3, x-1] \cup[x+2, x+4]$ we have $\operatorname{gcd}(z, n)=1$. Hence for every $t \in\left[0,\left\lfloor\frac{s}{2}\right\rfloor-1\right]$

$$
([x-3, x-1] \cup[x+2, x+4]) \cap\left[\frac{(2 s-2 t-1) n}{2 b}, \frac{(s-t) n}{b}\right]=\emptyset .
$$

If $t=0$, then $[x-3, x-1] \subset\left[\frac{(s-1) n}{b}, \frac{(2 s-1) n}{2 b}\right]$. Then $\frac{n}{2 b}=\frac{(2 s-1) n}{2 b}-\frac{(s-1) n}{b}>2$. Hence $\left[\frac{(2 s-3) n}{2 b}, \frac{(s-1) n}{b}\right]$ contains at least two integers. It follows from (i) that $\left[\frac{(s-1) n}{b}, \frac{(2 s-1) n}{2 b}\right]$ contains at most three integers, so we have that $x-4, x-5 \in\left[\frac{(2 s-3) n}{2 b}, \frac{(s-1) n}{b}\right]$. Then $\operatorname{gcd}(x-4, n)>1$ and 
$\operatorname{gcd}(x-5, n)>1$, which together with $\operatorname{gcd}(x, n)>1$ and $\operatorname{gcd}(x+1, n)>1$ yield a contradiction to the assumption that $n=p_{1}^{\alpha} \cdot p_{2}^{\beta}$ and $\operatorname{gcd}(n, 6)=1$. If $t \geq 1$, similarly, we can show that $x+5, x+6 \in\left[\frac{(2 s-2 t+1) n}{2 b}, \frac{(s-t+1) n}{b}\right]$ and thus $\operatorname{gcd}(x+5, n)>1$ and $\operatorname{gcd}(x+6, n)>1$, which yield a contradiction again. Hence, $\left[\frac{(2 s-2 t-1) n}{2 b}, \frac{(s-t) n}{b}\right]$ must contain exactly one integer for every $t \in\left[0,\left\lfloor\frac{s}{2}\right\rfloor-1\right]$, and therefore, $\frac{n}{2 b}=\frac{(s-t) n}{b}-\frac{(2 s-2 t-1) n}{2 b}<2$.

(iii). Since the length of $\left[\frac{(s-t) n}{b}, \frac{(2 s-2 t+1) n}{2 b}\right]$ is less than 2 by (ii), this interval contains at most two integers for each $t \in\left[1,\left\lfloor\frac{s-1}{2}\right\rfloor\right]$. Since $x \in\left[\frac{(2 s-2 t-1) n}{2 b}, \frac{(s-t) n}{b}\right]$ and $y \in\left[\frac{(2 s-2 t-3) n}{2 b}, \frac{(s-t-1) n}{b}\right]$, we have $\operatorname{gcd}(x, n)>1$, and $\operatorname{gcd}(y, n)>1$. Note that $2 \leq x-y \leq 3$ and $\operatorname{gcd}(n, 6)=1$. We infer that $\operatorname{gcd}(n, x-y)=1$ and thus $\operatorname{gcd}(x, y, n)=1$. This proves (iii).

(iv). Assume that $s \geq 6$, and then $\left\lfloor\frac{s}{2}\right\rfloor-1 \geq 2$. Assume that $x \in\left[\frac{(2 s-2 t-1) n}{2 b}, \frac{(s-t) n}{b}\right], y \in$ $\left[\frac{(2 s-2 t-3) n}{2 b}, \frac{(s-t-1) n}{b}\right]$ and $z \in\left[\frac{(2 s-2 t-5) n}{2 b}, \frac{(s-t-2) n}{b}\right]$. Then $\operatorname{gcd}(x, n)>1, \operatorname{gcd}(y, n)>1$ and $\operatorname{gcd}(z, n)>1$. By (iii) we have $\operatorname{gcd}(x, y, n)=\operatorname{gcd}(y, z, n)=1$. Since $n=p_{1}^{\alpha} \cdot p_{2}^{\beta}$, we have that $\operatorname{gcd}(x, z, n)=p_{1}^{\alpha_{1}}$ or $p_{2}^{\beta_{1}}$, where $1 \leq \alpha_{1} \leq \alpha$ and $1 \leq \beta_{1} \leq \beta$. Since $\operatorname{gcd}(n, 6)=1$ and $3 \leq x-z \leq 6$, we have that $\operatorname{gcd}(x-z, n)=x-z=5$ and thus $5 \mid \operatorname{gcd}(x, z, n)$. Note that $\left[\frac{(2 s-5) n}{2 b}, \frac{s n}{b}\right]$ contains exactly 6 integers, so we infer that $\frac{s n}{b}-\frac{(2 s-5) n}{2 b}<7$. Hence $\frac{n}{2 b}<\frac{7}{5}$, proving (iv).

(v). Assume to the contrary that $s \geq 8$. Then $\left\lfloor\frac{s}{2}\right\rfloor-1 \geq 3$. Assume that $x \in\left[\frac{(2 s-2 t-1) n}{2 b}, \frac{(s-t) n}{b}\right]$, $y \in\left[\frac{(2 s-2 t-3) n}{2 b}, \frac{(s-t-1) n}{b}\right], z \in\left[\frac{(2 s-2 t-5) n}{2 b}, \frac{(s-t-2) n}{b}\right]$ and $w \in\left[\frac{(2 s-2 t-7) n}{2 b}, \frac{(s-t-3) n}{b}\right]$. By (iv) we have $5 \mid x$ and $5 \mid y$, which is impossible since $2 \leq x-y \leq 3$.

This completes the proof.

Lemma 2.7. Proposition 2.2 holds if $a=2$.

Proof. Note that $S=g((b+1) g)((n-b) g)((n-2) g)$. By Lemmas 2.5 and 2.6, we may assume that $s \leq 7$, and thus $b<(s+1) a \leq 16$. Let $n=r b+b_{0}$, where $0 \leq b_{0} \leq b-1$. Then $n<(r+1) b$ and thus $r>\frac{n}{b}-1 \geq \frac{1000}{16}-1>60$.

If $b=2 t$, then let $m=(n-1) / 2$, and clearly $\operatorname{gcd}(m, n)=1$. Since $|m|_{n}+|m(b+1)|_{n}+\mid m(n-$ b) $\left.\right|_{n}+|m(n-2)|_{n}=m+(m-t)+t+1=n$, we have $\operatorname{ind}(S)=1$.

If $b=2 t+1$, since $b \geq a=2$, we have $1 \leq t<\frac{b}{2}<\frac{n}{4}$. Take $m_{1}=\frac{n-1}{2}$. Then $\left\{\left|m_{1}\right|_{n}, \mid m_{1}(n-\right.$ $\left.2)\left.\right|_{n},\left|m_{1}(n-b)\right|_{n},\left|m_{1}(b+1)\right|_{n}\right\}=\left\{1, \frac{n-1}{2}, n-\frac{n-b}{2}, n-t-1\right\}$, and $1<t+1<\frac{n-b}{2}<\frac{n-1}{2}$. Let $S^{\prime}=g\left(c^{\prime} g\right)\left(\left(n-b^{\prime}\right) g\right)\left(\left(n-a^{\prime}\right) g\right)$, where $a^{\prime}=t+1, b^{\prime}=\frac{n-b}{2}$ and $c^{\prime}=\frac{n-1}{2}$. Since $\operatorname{gcd}\left(m_{1}, n\right)=1$ we have $\operatorname{ind}(S)=\operatorname{ind}\left(S^{\prime}\right)$, and we shall show that $\operatorname{ind}\left(S^{\prime}\right)=1$.

Take $k^{\prime}=\left\lceil\frac{n-b}{2 b}\right\rceil$. It is easy to verify that $\frac{\left(k^{\prime}+i\right) n}{c^{\prime}} \leq 2\left(k^{\prime}+i\right)+1 \leq \frac{\left(k^{\prime}+i\right) n}{b^{\prime}}$ for each $i \in[0,2]$. Since $\operatorname{gcd}(n, 6)=1$ and $n=p_{1}^{\alpha} \cdot p_{2}^{\beta}$, we have $\operatorname{gcd}\left(2\left(k^{\prime}+i\right)+1, n\right)=1$ for some $i \in[0,2]$. Now let $m$ be one of the integers in $\left\{2 k^{\prime}+1,2 k^{\prime}+3,2 k^{\prime}+5\right\}$ which is co-prime to $n$ and let $k=\frac{m-1}{2}$. Then $k \leq b^{\prime}$. We shall show that $m a^{\prime}<n$, and then the result follows from Lemma 2.3 (1).

If $r \equiv 1(\bmod 2)$, then $k^{\prime}=\left\lceil\frac{n-b}{2 b}\right\rceil=\frac{r+1}{2}$. Since $r>60$, we have that $m a^{\prime} \leq\left(2 k^{\prime}+5\right)(t+1)=$ $(r+6)(t+1)<r(2 t+1)+b_{0}$ and we are done.

If $r \equiv 0(\bmod 2)$, then $k^{\prime}=\left\lceil\frac{n-b}{2 b}\right\rceil=\frac{r}{2}$. Since $r>60$, we have that $m a^{\prime} \leq\left(2 k^{\prime}+5\right)(t+1)=$ $(r+5)(t+1)<r(2 t+1)+b_{0}$, and we are done. 


\section{Proof of Theorem 1.3}

As mentioned in the last section, we need only prove Proposition 2.2. In view of Lemmas 2.5, 2.6 and 2.7 from now on we may always assume that $s \leq 7$ and $a \geq 3$.

Let $k_{1}$ be the largest positive integer such that $\left\lceil\frac{\left(k_{1}-1\right) n}{c}\right\rceil=\left\lceil\frac{\left(k_{1}-1\right) n}{b}\right\rceil$ and $\frac{k_{1} n}{c} \leq m<\frac{k_{1} n}{b}$. Since $\frac{b n}{c} \leq n-1<n=\frac{b n}{b}$ and $\frac{t n}{b}-\frac{t n}{c}=\frac{t(c-b) n}{b c}>2$ for all $t \geq b$, such integer $k_{1}$ always exists and $k_{1} \leq b$. Since $\left\lceil\frac{\left(k_{1}-1\right) n}{c}\right\rceil=\left\lceil\frac{\left(k_{1}-1\right) n}{b}\right\rceil$, we have

$$
1>\frac{\left(k_{1}-1\right) n}{b}-\frac{\left(k_{1}-1\right) n}{c}=\frac{\left(k_{1}-1\right) n(c-b)}{b c}=\frac{\left(k_{1}-1\right) n(a-1)}{b c} .
$$

We now show that Proposition 2.2 holds through the following 3 propositions. The first one handles the case when $\left\lceil\frac{n}{c}\right\rceil<\left\lceil\frac{n}{b}\right\rceil\left(\right.$ i.e. $k_{1}=1$ ), and the others handle the case when $\left\lceil\frac{n}{c}\right\rceil=\left\lceil\frac{n}{b}\right\rceil\left(\right.$ i.e. $\left.k_{1} \geq 2\right)$.

Proposition 3.1. Suppose $\left\lceil\frac{n}{c}\right\rceil<\left\lceil\frac{n}{b}\right\rceil$ (i.e. there exists a positive integer $m_{1}$ such that $\frac{n}{c} \leq$ $\left.m_{1}<\frac{n}{b}\right)$, then Proposition 2.2 holds.

Proposition 3.2. Suppose $\left\lceil\frac{n}{c}\right\rceil=\left\lceil\frac{n}{b}\right\rceil$. Let $k_{1}$ be the largest positive integer such that $\left\lceil\frac{\left(k_{1}-1\right) n}{c}\right\rceil=$ $\left\lceil\frac{\left(k_{1}-1\right) n}{b}\right\rceil$ and $\frac{k_{1} n}{c} \leq m_{1}<\frac{k_{1} n}{b}$ holds for some integer $m_{1}$. If $k_{1}>\frac{b}{a}$, then Proposition 2.2 holds.

Proposition 3.3. Suppose $\left\lceil\frac{n}{c}\right\rceil=\left\lceil\frac{n}{b}\right\rceil$. Let $k_{1}$ be the largest positive integer such that $\left\lceil\frac{\left(k_{1}-1\right) n}{c}\right\rceil=$ $\left\lceil\frac{\left(k_{1}-1\right) n}{b}\right\rceil$ and $\frac{k_{1} n}{c} \leq m_{1}<\frac{k_{1} n}{b}$ holds for some integer $m_{1}$. If $k_{1} \leq \frac{b}{a}$, then Proposition 2.2 holds.

\subsection{Proof of Proposition 3.1}

In this subsection, we assume that $\left\lceil\frac{n}{c}\right\rceil<\left\lceil\frac{n}{b}\right\rceil$. Let $m_{1}=\left\lceil\frac{n}{c}\right\rceil$. Then we have $m_{1}-1<\frac{n}{c} \leq$ $m_{1}<\frac{n}{b}$. By Lemma 2.3 (1), it suffices to find $k$ and $m$ such that $\frac{k n}{c} \leq m<\frac{k n}{b}, \operatorname{gcd}(m, n)=$ $1,1 \leq k \leq b$, and $m a<n$. So in what follows, we may always assume that $\operatorname{gcd}\left(n, m_{1}\right)>1$.

Lemma 3.4. If $\left[\frac{n}{c}, \frac{n}{b}\right]$ contains at least two integers, then $\operatorname{ind}(S)=1$.

Proof. Since $a \leq b$, by Lemma 2.4 we may assume every integer in $\left[\frac{n}{c}, \frac{n}{b}\right]$ is not co-prime to $n$. Since $n=p_{1}^{\alpha} \cdot p_{2}^{\beta}$, we may assume that $\left[\frac{n}{c}, \frac{n}{b}\right]$ contains exactly two integers. Then

$$
m_{1}-1<\frac{n}{c} \leq m_{1}<m_{1}+1 \leq \frac{n}{b}<m_{1}+2,
$$

and we also have that $\operatorname{gcd}\left(n, m_{1}\right)>1$ and $\operatorname{gcd}\left(n, m_{1}+1\right)>1$. Since $n=p_{1}^{\alpha} \cdot p_{2}^{\beta}$ and $\operatorname{gcd}(n, 6)=1$, we infer that $m_{1} \geq 10$ and $\operatorname{gcd}\left(2 m_{1}+1, n\right)=1$. Then $n \geq\left(m_{1}+1\right) b \geq 11 b$.

Note that

$$
2 m_{1}-2<\frac{2 n}{c} \leq 2 m_{1}<2 m_{1}+1<2 m_{1}+2 \leq \frac{2 n}{b}<2 m_{1}+4 .
$$

Let $m=2 m_{1}+1$ and $k=2$. We shall show that $m a<n$. 
Since $1+c=a+b$, by (3.2) we have $\left(2 m_{1}-2\right)(b+a-1)=\left(2 m_{1}-2\right) c<2 n<\left(2 m_{1}+4\right) b$, and thus $\left(2 m_{1}-2\right)(a-1)<6 b$. Since $a \geq 3$ and $m_{1} \geq 10$, we have

$$
m a=\left(2 m_{1}+1\right) a=\frac{2 m_{1}+1}{2 m_{1}-2} \times \frac{a}{a-1} \times\left(2 m_{1}-2\right)(a-1)<\frac{2 \times 10+1}{2 \times 10-2} \times \frac{3}{3-1} \times 6 b<11 b \leq n,
$$

and we are done.

By Lemma 3.4, we may assume that $\left[\frac{n}{c}, \frac{n}{b}\right]$ contains exactly one integer $m_{1}$, and thus

$$
m_{1}-1<\frac{n}{c} \leq m_{1}<\frac{n}{b}<m_{1}+1 .
$$

Since $\operatorname{gcd}(n, 6)=1$ and $\left(n, m_{1}\right)>1$, we have either $m_{1}=5$ or $m_{1}=7$ or $m_{1} \geq 10$.

Let $\ell$ be the smallest integer such that $\left[\frac{\ell n}{c}, \frac{\ell n}{b}\right)$ contains at least three integers. Clearly, $\ell \geq 2$. Since $\frac{n}{b}-m_{1}<1$ and $m_{1}-\frac{n}{c}<1$, by using the minimality of $\ell$ we obtain that $\ell m_{1}-3<\frac{\ell n}{c}<\frac{\ell n}{b}<\ell m_{1}+3$. Then $\frac{\ell n(c-b)}{b c}=\frac{\ell n}{b}-\frac{\ell n}{c}<\left(\ell m_{1}+3\right)-\left(\ell m_{1}-3\right)<6$ and thus $\ell<\frac{6 b c}{(c-b) n}=\frac{6 b}{c-b} \times \frac{c}{n}<\frac{6 b}{(a-1)\left(m_{1}-1\right)} \leq \frac{6 b}{(3-1)(5-1)}<b$.

We claim that $\left[\frac{\ell n}{c}, \frac{\ell n}{b}\right)$ contains at most four integers. Assume to the contrary that $\left[\frac{\ell n}{c}, \frac{\ell n}{b}\right)$ contains at least five integers, so $\ell m_{1}-3<\frac{\ell n}{c} \leq \ell m_{1}-2<\ell m_{1}+2<\frac{\ell n}{b} \leq \ell m_{1}+3$. Then $\frac{2}{\ell} \leq m_{1}-\frac{n}{c}, \frac{n}{b}-m_{1} \leq \frac{3}{\ell}$. Since $\ell \geq 2$, we have $(\ell-1)\left(m_{1}-\frac{n}{c}\right) \geq(\ell-1) \times \frac{2}{\ell} \geq 1$ and $(\ell-1)\left(\frac{n}{b}-m_{1}\right)>(\ell-1) \times \frac{2}{\ell} \geq 1$. Hence, $\frac{(\ell-1) n}{c} \leq(\ell-1) m_{1}-1<(\ell-1) m_{1}+1<\frac{(\ell-1) n}{b}$, so $\left[\frac{(\ell-1) n}{c}, \frac{(\ell-1) n}{b}\right)$ contains at least three integers, a contradiction to the minimality of $\ell$.

By the above claim we have either

$$
\ell m_{1}-2<\frac{\ell n}{c}<\frac{\ell n}{b}<\ell m_{1}+3
$$

or

$$
\ell m_{1}-3<\frac{\ell n}{c}<\frac{\ell n}{b} \leq \ell m_{1}+2 .
$$

We remark that since $n=p_{1}^{\alpha} \cdot p_{2}^{\beta}$ and $\left[\frac{\ell n}{c}, \frac{\ell n}{b}\right)$ contains at least 3 integers, one of them (say $m$ ) must be co-prime to $n$. If $m a<n$, then we are done by Lemma 2.3 (1) (with $k=l<b$ ). Otherwise, for each integer $\delta \in[1, \ell-1],\left[\frac{\delta n}{c}, \frac{\delta n}{b}\right)$ contains at most 2 integers. We shall try to find an integer $m$ in one of those intervals such that $m a<n$ and this method will be used frequently in sequel.

Recall that $\left[\frac{n}{c}, \frac{n}{b}\right]$ contains exactly one integer $m_{1}, \operatorname{gcd}\left(m_{1}, n\right)>1$ and $\ell \geq 2$. We first deal with two special cases in the following two lemmas. More specifically, we shall show that if $\left[\frac{n}{c}, \frac{n}{b}\right]$ contains 5 or 7 , then Proposition 3.1 holds.

Lemma 3.5. If $4<\frac{n}{c} \leq 5<\frac{n}{b}<6$ and $5 \mid n$, then $\operatorname{ind}(S)=1$.

Proof. Since $4<\frac{n}{c} \leq 5<\frac{n}{b}<6, n>5 b$. Note that $m_{1}=\left\lceil\frac{n}{c}\right\rceil=5$.

If $\ell=2$, since $\left[\frac{\ell n}{c}, \frac{\ell n}{b}\right)$ contains at least three integers, we must have $8<\frac{2 n}{c}<9<10<$ $11<\frac{2 n}{b}<12$. Thus $\frac{n}{6}<b<c<\frac{n}{4}$. Let $m=9$ and $k=2$. Then $9 a=9 \times(c-b+1) \leq$ $9 \times\left(\frac{n-1}{4}-\frac{n+1}{6}+1\right)<n$, and we are done.

Next assume that $\ell \geq 3$. Since $\left[\frac{\ell n}{c}, \frac{\ell n}{b}\right)$ contains at least three integers and $5 \ell-3<\frac{\ell n}{c}<\frac{\ell n}{b} \leq$ $5 \ell+3$, we can divide the proof into three cases. 
Case 1. $5 \ell+2 \leq \frac{\ell n}{b}<5 \ell+3$. Then $\frac{2}{\ell} \leq \frac{n}{b}-5 \leq \frac{3}{\ell}$.

If $\frac{\ell+1}{2} \leq \gamma \leq \ell-1$, then $\gamma\left(\frac{n}{b}-5\right)>\frac{\ell}{2} \cdot \frac{2}{\ell}=1$ and thus $\frac{\gamma n}{c} \leq 5 \gamma<5 \gamma+1<\frac{\gamma n}{b}$. By the minimality of $\ell$ we infer that

$$
5 \gamma-1<\frac{\gamma n}{c} \leq 5 \gamma<5 \gamma+1<\frac{\gamma n}{b} \leq 5 \gamma+2 .
$$

Let $\gamma=\ell-1$. By (3.5) , we have $(5(\ell-1)-1)(b+a-1)=(5 \ell-6) c<(\ell-1) n \leq(5(\ell-1)+2) b$ and thus $(5 \ell-6)(a-1)<3 b$.

First assume that $\ell \geq 16$. Let $k=\ell$ and let $m$ be an integer in $\left[\frac{\ell n}{c}, \frac{\ell n}{b}\right)$ which is co-prime to $n$. Since $5 \ell+2 \leq \frac{\ell n}{b}<5 \ell+3$, we have that $m \leq 5 \ell+2$. Then

$$
m a \leq(5 \ell+2) a=\frac{5 \ell+2}{5 \ell-6} \times \frac{a}{a-1} \times(5 \ell-6)(a-1)<\frac{5 \times 16+2}{5 \times 16-6} \times \frac{3}{3-1} \times 3 b<5 b \leq n,
$$

and we are done.

Next assume that $6 \leq \ell \leq 15$.

If $\operatorname{gcd}(5(\ell-1)+1, n)=1$, let $m=5(\ell-1)+1$ and $k=\ell-1$. Then by (3.5) $\frac{k n}{c}<m<\frac{k n}{b}$ and

$$
(5(\ell-1)+1) a=\frac{5(\ell-1)+1}{5 \ell-6} \times \frac{a}{a-1} \times(5 \ell-6)(a-1)<\frac{5 \times 6-4}{5 \times 6-6} \times \frac{3}{3-1} \times 3 b<5 b \leq n,
$$

as desired. Thus we may assume that $\operatorname{gcd}(5(\ell-1)+1, n)>1$.

If $13 \leq \ell \leq 15$, applying (3.5) with $\gamma=8$, we have $39<\frac{8 n}{c}<40<41<\frac{8 n}{b} \leq 42$. Thus $\frac{4 n}{21} \leq b<c<\frac{8 n}{39}$. Since $\operatorname{gcd}(5(\ell-1)+1, n)>1$ and $\operatorname{gcd}(5, n)>1$ and $n=p_{1}^{\alpha} \cdot p_{2}^{\beta}$, we have that $\operatorname{gcd}(41, n)=1$. Let $m=41$ and $k=8$. Then $41 a=41(c-b+1)<41\left(\frac{8 n}{39}-\frac{4 n}{21}+1\right)<n$, and we are done.

If $8 \leq \ell \leq 12$, applying (3.5) with $\gamma=7$, we have $34<\frac{7 n}{c}<35<36<\frac{7 n}{b} \leq 37$, and so $\frac{7 n}{37} \leq b<c<\frac{7 n}{34}$. Note that $\operatorname{gcd}(36, n)=1$. Let $m=36$ and $k=7$. Then $36 a=36 \times(c-b+1)<$ $36 \times\left(\frac{7 n}{34}-\frac{7 n}{37}+1\right)<n$, and we are done.

If $6 \leq \ell \leq 7$, applying (3.5) with $\gamma=4$, we have $19<\frac{4 n}{c}<20<21<\frac{4 n}{b} \leq 22$, and so $\frac{2 n}{11} \leq b<c<\frac{4 n}{19}$. As above we have $\operatorname{gcd}(21, n)=1$. Let $m=21$ and $k=4$. Then $21 a=21(c-b+1)<21\left(\frac{4 n}{19}-\frac{2 n}{11}+1\right)<n$, and we are done.

Finally, assume that $\ell \leq 5$.

If $4 \leq \ell \leq 5$, applying (3.5) with $\gamma=3$, we have $14<\frac{3 n}{c}<15<16<\frac{3 n}{b} \leq 17$, then $\frac{3 n}{17}<b<c<\frac{3 n}{14}$. Note that $\operatorname{gcd}(16, n)=1$. Now let $m=16$ and $k=3$. Then $16 a=$ $16(c-b+1)<16\left(\frac{3 n}{14}-\frac{3 n}{17}+1\right)<n$, and we are done.

If $\ell=3$, we have $\frac{3 n}{c}<15<16<17<\frac{3 n}{b} \leq 18$. If $\frac{3 n}{c} \geq 14$, then $c \leq \frac{3 n}{14}$ and $b \geq \frac{n}{6}$. Note that $\operatorname{gcd}(16, n)=1$. Let $m=16$ and $k=3$. Then $16 a=16 \times(c-b+1) \leq 16 \times\left(\frac{3 n}{14}-\frac{n}{6}+1\right)<n$, and we are done. Now assume that $\frac{3 n}{c}<14$, by (3.3) we have $13<\frac{3 n}{c}<14$. Applying (3.5) with $\gamma=2$, we have $9<\frac{2 n}{c}<10<11<\frac{2 n}{b} \leq 12$, and then $\frac{n}{6}<b<c<\frac{2 n}{9}$. Note that either $\operatorname{gcd}(11, n)=1$ or $\operatorname{gcd}(14, n)=1$. Now let $k=2$ and $m=11$ if $\operatorname{gcd}(m, 11)=1$, or let $k=3$ and $m=14$ if $\operatorname{gcd}(m, 14)=1$. Then $m a \leq 14 \times(c-b+1)<14 \times\left(\frac{2 n}{9}-\frac{n}{6}+1\right)<n$.

This completes the proof of Case 1 .

Case 2. $\frac{\ell n}{b}<5 \ell+2$ and $5 \ell-3<\frac{\ell n}{c} \leq 5 \ell-2$. This case can be proved in a similar way to Case 1. 
Case 3. $\frac{\ell n}{b}<5 \ell+2$ and $\frac{\ell n}{c}>5 \ell-2$. Thus $5 \ell-2<\frac{\ell n}{c} \leq 5 \ell-1<5 \ell<5 \ell+1<\frac{\ell n}{b}<5 \ell+2$. This implies that every integer in $\left[\frac{\ell n}{c}, \frac{\ell n}{b}\right)$ is less than $5 \ell+2$. By the minimality of $\ell$, we must have one of the following holds.

(i) $5(\ell-1)-1<\frac{(\ell-1) n}{c}<5(\ell-1)<\frac{(\ell-1) n}{b} \leq 5(\ell-1)+1$.

(ii) $5(\ell-1)-1<\frac{(\ell-1) n}{c}<5(\ell-1)<5(\ell-1)+1<\frac{(\ell-1) n}{b}<5(\ell-1)+2$.

(iii) $5(\ell-1)-2<\frac{(\ell-1) n}{c} \leq 5(\ell-1)-1<5(\ell-1)<\frac{(\ell-1) n}{b} \leq 5(\ell-1)+1$.

We divide the proof into three subcases according to the above three situations.

Subcase 3.1. (i) holds. Then $(5(\ell-1)-1)(b+a-1)=(5(\ell-1)-1) c<(\ell-1) n \leq(5(\ell-1)+1) b$, so $(5 \ell-6)(a-1)<2 b$.

If $\ell \geq 4$, let $k=\ell$ and $m$ be an integer in $\left[\frac{\ell n}{c}, \frac{\ell n}{b}\right)$ which is co-prime to $n$. Note that $m \leq 5 \ell+1$. Then

$$
m a \leq(5 \ell+1) a=\frac{5 \ell+1}{5 \ell-6} \times \frac{a}{a-1} \times(5 \ell-6)(a-1)<\frac{5 \times 4+1}{5 \times 4-6} \times \frac{3}{3-1} \times 2 b<5 b \leq n,
$$

so we are done. Therefore, we may assume that $\ell=3$, so $13<\frac{3 n}{c}<14<15<16<\frac{3 n}{b}<17$. Since $9<\frac{2 n}{c}<10<\frac{2 n}{b}<11$, we have $\frac{2 n}{11}<b<c<\frac{2 n}{9}$. Let $m=16$ and $k=3$. Then $m a=16 \times(c-b+1)<16 \times\left(\frac{2 n}{9}-\frac{2 n}{11}+1\right)<n$, and we are done.

Subcase 3.2. (ii) holds. Then $(5(\ell-1)-1)(b+a-1)=(5(\ell-1)-1) c<(\ell-1) n<(5(\ell-1)+2) b$, so $(5 \ell-6)(a-1)<3 b$.

If $\ell \geq 14$, then let $k=\ell$ and let $m$ be an integer in $\left[\frac{\ell n}{c}, \frac{\ell n}{b}\right)$ which is co-prime to $n$. Note that $m \leq 5 \ell+1$. Thus

$$
m a \leq(5 \ell+1) a=\frac{5 \ell+1}{5 \ell-6} \times \frac{a}{a-1} \times(5 \ell-6)(a-1)<\frac{5 \times 14+1}{5 \times 14-6} \times \frac{3}{3-1} \times 3 b<5 b \leq n,
$$

and we are done.

Next assume that $5 \leq \ell \leq 13$. If $\operatorname{gcd}(5(\ell-1)+1, n)=1$, let $m=5(\ell-1)+1$ and $k=\ell-1$. Then

$$
m a=(5(\ell-1)+1) a=\frac{5(\ell-1)+1}{5 \ell-6} \times \frac{a}{a-1} \times(5 \ell-6)(a-1)<\frac{5 \times 5-4}{5 \times 5-6} \times \frac{3}{3-1} \times 3 b<5 b \leq n,
$$

and we are done. Hence we may assume that $\operatorname{gcd}(5(\ell-1)+1, n)>1$, which together with $\operatorname{gcd}(5, n)>1$ and $n=p_{1}^{\alpha} \cdot p_{2}^{\beta}, \operatorname{implies} \operatorname{gcd}(5 \ell-1, n)=1$. Now let $m=5 \ell-1$ and $k=\ell$. Since $\frac{\ell n}{5 \ell+2}<b<c<\frac{\ell n}{5 \ell-2}$, we have $m a=(5 \ell-1)(c-b+1)<(5 \ell-1)\left(\frac{\ell n}{5 \ell-2}-\frac{\ell n}{5 \ell+2}+1\right)<n$, and we are done.

Finally, assume that $\ell \leq 4$.

If $\ell=4$, then $14<\frac{3 n}{c}<15<16<\frac{3 n}{b}<17$. It follows that $\frac{3 n}{17}<b<c<\frac{3 n}{14}$. Note that $\operatorname{gcd}(16, n)=1$. Let $m=16$ and $k=3$. Then $m a=16 \times(c-b+1) \leq 16 \times\left(\frac{3 n-1}{14}-\frac{3 n+1}{17}+1\right)<n$, and we are done.

If $\ell=3$, we have $9<\frac{2 n}{c}<10<11<\frac{2 n}{b}<12$ and $13<\frac{3 n}{c}<14<15<\frac{3 q}{b}<18$. Hence $\frac{n}{6}<b<c<\frac{2 n}{9}$. Now let $m=11$ and $k=2$ if $\operatorname{gcd}(11, n)=1$ or let $m=14$ and $k=3$ if $\operatorname{gcd}(n, 14)=1$. Then $m a \leq 14 a=14 \times(c-b+1) \leq 14 \times\left(\frac{2 n-1}{9}-\frac{n+1}{6}+1\right)<n$, and we are done.

Subcase 3.3. (iii) holds. This subcase can be proved in a similar way to Subcase 3.2. 
Lemma 3.6. If $6<\frac{n}{c} \leq 7<\frac{n}{b}<8$ and $7 \mid n$, then $\operatorname{ind}(S)=1$.

Proof. Since $6<\frac{n}{c} \leq 7<\frac{n}{b}<8, n>7 b$. Note that $m_{1}=7$.

If $\ell=2$, then $12<\frac{2 n}{c} \leq 13<14<15<\frac{2 n}{b} \leq 16$. Since $12(b+a-1)=12 c<2 n \leq 16 b$, we have that $12(a-1)<4 b$. If $\operatorname{gcd}(15, n)=1$, let $m=15$ and $k=2$; otherwise let $m=13$ and $k=2$. Then

$$
m a \leq 15 a=\frac{15}{12} \times \frac{a}{a-1} \times 12(a-1)<\frac{15}{12} \times \frac{3}{3-1} \times 4 b<7 b<n,
$$

and we are done.

Next assume that $\ell \geq 3$. Recall that $7 \ell-3<\frac{\ell n}{c} \leq 7 \ell<\frac{\ell n}{b}<7 \ell+3$. We distinguish three cases according to where $\frac{\ell n}{c}$ locates.

Case 1. $7 \ell-1<\frac{\ell n}{c} \leq 7 \ell$.

Then $7 \ell-1<\frac{\ell n}{c} \leq 7 \ell<7 \ell+1<7 \ell+2<\frac{\ell n}{b} \leq 7 \ell+3$. It follows that $(7 \ell-1)(b+a-1)=$ $(7 \ell-1) c<\ell n \leq(7 \ell+3) b$, hence $(7 \ell-1)(a-1)<4 b$. If $\operatorname{gcd}(7 \ell+2, n)>1$, then $\operatorname{gcd}(7 \ell+1, n)=1$, so let $m=7 \ell+1$ and $k=\ell$; otherwise let $m=7 \ell+2$ and $k=\ell$. Then

$$
m a \leq(7 \ell+2) a=\frac{7 \ell+2}{7 \ell-1} \times \frac{a}{a-1} \times(7 \ell-1)(a-1)<\frac{7 \times 3+2}{7 \times 3-1} \times \frac{3}{3-1} \times 4 b<7 b \leq n,
$$

and we are done.

Case 2. $7 \ell-2<\frac{\ell n}{c} \leq 7 \ell-1$. Since $\left[\frac{\ell n}{c}, \frac{\ell n}{b}\right)$ contains at most four integers, we distinguish two subcases.

Subcase 2.1. $\left[\frac{\ell n}{c}, \frac{\ell n}{b}\right)$ contains four integers.

Then $7 \ell-2<\frac{\ell n}{c} \leq 7 \ell-1<7 \ell<7 \ell+2 \leq \frac{\ell n}{b} \leq 7 \ell+3$. It follows that $\frac{1}{\ell} \leq 7-\frac{n}{c}<\frac{2}{\ell}<\frac{n}{b}-7 \leq \frac{3}{\ell}$. Therefore, $(\ell-1)\left(\frac{n}{b}-7\right)>(\ell-1) \frac{2}{\ell}>1$. By the minimality of $\ell$ we have

$$
7(\ell-1)-1<\frac{(\ell-1) n}{c}<7(\ell-1)<7(\ell-1)+1<\frac{(\ell-1) n}{b} \leq 7(\ell-1)+2 .
$$

Then $(7 \ell-8)(b+a-1)=(7 \ell-8) c<(\ell-1) n \leq(7 \ell-5) b$; hence $(7 \ell-8)(a-1)<3 b$.

First assume that $\ell \geq 4$. If $\operatorname{gcd}(7 \ell+1, n)>1$, then $\operatorname{gcd}(7 \ell-1, n)=1$, so let $m=7 \ell-1$ and $k=\ell$; otherwise let $m=7 \ell+1$ and $k=\ell$.

$$
m a \leq(7 \ell+1) a=\frac{7 \ell+1}{7 \ell-8} \times \frac{a}{a-1} \times(7 \ell-8)(a-1)<\frac{7 \times 4+2}{7 \times 4-8} \times \frac{3}{3-1} \times 3 b<7 b \leq n,
$$

and we are done.

Next assume that $\ell=3$. Then $13<\frac{2 n}{c}<14<15<\frac{2 n}{b} \leq 16$. Note that $\frac{4 n}{c}<27<28<\frac{4 n}{b}$, $\operatorname{gcd}(27, n)=1$ and $\frac{n}{8}<b<c<\frac{2 n}{13}$. Let $m=27$ and $k=4$. Then $m a=27 \times(c-b+1) \leq$ $27 \times\left(\frac{2 n-1}{13}-\frac{n+1}{8}+1\right)<n$, and we are done.

Subcase 2.2. $\left[\frac{\ell n}{c}, \frac{\ell n}{b}\right)$ contains three integers.

Then $7 \ell-2<\frac{\ell n}{c} \leq 7 \ell-1<7 \ell<7 \ell+1<\frac{\ell n}{b} \leq 7 \ell+2$. It follows that $(7 \ell-2)(b+a-1)=$ $(7 \ell-2) c<\ell n \leq(7 \ell+2) b$; hence $(7 \ell-2)(a-1)<4 b$.

First assume that $\ell \geq 4$. If $\operatorname{gcd}(7 \ell+1, n)>1$, then $\operatorname{gcd}(7 \ell-1, n)=1$, so let $m=7 \ell-1$ and $k=\ell$; otherwise let $m=7 \ell+1$ and $k=\ell$. Then

$$
m a \leq(7 \ell+1) a=\frac{7 \ell+1}{7 \ell-2} \times \frac{a}{a-1} \times(7 \ell-2)(a-1)<\frac{7 \times 4+2}{7 \times 4-2} \times \frac{3}{3-1} \times 4 b<7 b \leq n,
$$

and we are done. 
Next assume that $\ell=3$. Then $19<\frac{3 n}{c}<20<21<22<\frac{3 n}{b} \leq 23$. If $\operatorname{gcd}(n, 20)=1$, let $k=3$ and $m=20$; otherwise let $m=22$ and $k=3$. Note that $\frac{3 n}{23}<b<c<\frac{3 n}{19}$. Then $m a \leq 22 \times(c-b+1) \leq 22 \times\left(\frac{3 n-1}{19}-\frac{3 n+1}{23}+1\right)<n$, and we are done.

Case 3. $7 \ell-3<\frac{\ell n}{c} \leq 7 \ell-2$. As in Case 2, we distinguish two subcases.

Subcase 3.1. $\left[\frac{\ell n}{c}, \frac{\ell n}{b}\right)$ contains four integers.

It follows that $7 \ell-3<\frac{\ell n}{c} \leq 7 \ell-2<7 \ell<7 \ell+1 \leq \frac{\ell n}{b} \leq 7 \ell+2$. The proof is similar to Subcase 2.1.

Subcase 3.2. $\left[\frac{\ell n}{c}, \frac{\ell n}{b}\right)$ contains three integers.

It follows that $7 \ell<\frac{\ell n}{b}<7 \ell+1$. Similar to Case 1, We can prove the result.

This completes the proof.

Now we are in a position to prove Proposition 3.1 .

\section{Proof of Proposition 3.1.}

Recall that either $m_{1}=5$ or $m_{1}=7$ or $m_{1} \geq 10$. By Lemmas 3.5 and 3.6 we may assume $m_{1} \geq 10$. Then $n \geq m_{1} b \geq 10 b$. Let $k=\ell$ and let $m$ be one of the integers in $\left[\frac{\ell n}{c}, \frac{\ell n}{b}\right)$ which is co-prime to $n$. Recall that we have either (3.3) holds or (3.4) holds.

If (3.3) holds, then $\left(\ell m_{1}-2\right)(b+a-1)=\left(\ell m_{1}-2\right) c<\ell n \leq\left(\ell m_{1}+3\right) b$, so $\left(\ell m_{1}-2\right)(a-1)<5 b$. Note that $m \leq \ell m_{1}+2$ and $\ell \geq 2$, then

$$
m a \leq\left(\ell m_{1}+2\right) a=\frac{\ell m_{1}+2}{\ell m_{1}-2} \times \frac{a}{a-1} \times\left(\ell m_{1}-2\right)(a-1)<\frac{2 \times 10+2}{2 \times 10-2} \times \frac{3}{3-1} \times 5 b<10 b \leq n,
$$

and we are done.

If (3.4) holds, then $\left(\ell m_{1}-3\right)(b+a-1)=\left(\ell m_{1}-3\right) c<\ell n \leq\left(\ell m_{1}+2\right) b$, hence $\left(\ell m_{1}-3\right)(a-1)<$ $5 b$. Note that $m \leq \ell m_{1}+1$ and $\ell \geq 2$. Then

$$
m a \leq\left(\ell m_{1}+1\right) a=\frac{\ell m_{1}+1}{\ell m_{1}-3} \times \frac{a}{a-1} \times\left(\ell m_{1}-3\right)(a-1)<\frac{2 \times 10+1}{2 \times 10-3} \times \frac{3}{3-1} \times 5 b<10 b \leq n,
$$

and we are done.

\subsection{Proof of Proposition 3.2}

In this subsection, we always assume that $\left\lceil\frac{n}{c}\right\rceil=\left\lceil\frac{n}{b}\right\rceil$, so $k_{1} \geq 2$, and we also assume that $k_{1}>\frac{b}{a}$.

\section{Proof of Proposition 3.2}

We distinguish the proof to two cases.

Case 1. $k_{1}=2$.

If $a-1 \geq \frac{b}{2}$, then

$$
\frac{(c-b)\left(k_{1}-1\right) n}{c b}=\frac{(a-1) n}{b c}=\frac{a-1}{b} \times \frac{n}{c}>\frac{1}{2} \times 2=1,
$$

which is a contradiction to (3.1). Hence, $\frac{b}{2}-1<a-1<\frac{b}{2}$.

If $b=2 \ell$, then $\ell-1<a-1<\ell$, which is impossible. Therefore, we must have that $b=2 \ell+1$, and thus $a=\ell+1$. Now $c=a+b-1=3 \ell+1$ and $c-b=a-1=\ell$.

If $n \geq 3 c$, then

$$
\frac{(c-b)\left(k_{1}-1\right) n}{c b}=\frac{\ell n}{(2 \ell+1) c} \geq \frac{3 \ell}{2 \ell+1} \geq 1,
$$


which is a contradiction to (3.1).

Thus $n<3 c$, so we assume that $n=2 c+\ell_{0}$ for some $\ell_{0}$ odd $\operatorname{since} \operatorname{gcd}(n, 6)=1$. If $\ell_{0}=1$, then $n=2 c+1=2(3 \ell+1)+1=3(2 \ell+1)$, a contradiction (since $n$ is not divisible by 3 ). If $\ell_{0}=3$, then $n=2 c+3$ and thus $\frac{n}{c} \leq 3<\frac{n}{b}=\frac{6 \ell+5}{2 \ell+1}$. This implies that $\left\lceil\frac{n}{c}\right\rceil<\left\lceil\frac{n}{b}\right\rceil$, a contradiction. If $\ell_{0} \geq 5$, then $n \geq 2 c+5=6 \ell+7$. Now

$$
\frac{(c-b)\left(k_{1}-1\right) n}{c b}=\frac{\ell n}{(2 \ell+1)(3 \ell+1)} \geq \frac{\ell(6 \ell+7)}{6 \ell^{2}+5 \ell+1}>1,
$$

a contradiction to (3.1).

Case 2. $k_{1} \geq 3$.

If $a-1 \geq \frac{b}{k_{1}}$, then $\frac{(c-b)\left(k_{1}-1\right) n}{c b} \geq \frac{b\left(k_{1}-1\right) n}{c b k_{1}}>\frac{2\left(k_{1}-1\right)}{k_{1}}>1$, a contradiction.

Thus, we have that $\frac{b}{k_{1}}+1>a>\frac{b}{k_{1}}$. Assume that $b=k_{1} \ell+k_{0}$ for some $0 \leq k_{0}<k_{1}$ and $\ell \geq 1$. Note that if $k_{0}=0$, then $\ell+1>a>\frac{b}{k_{1}}=\ell$, a contradiction. Therefore, we have that $1 \leq k_{0}<k_{1}$. Then $a=\ell+1$, so $c=a+b-1=\left(k_{1}+1\right) \ell+k_{0}$ and also $c-b=a-1=\ell$. Now (3.1) reduces to the following.

$$
\frac{(c-b)\left(k_{1}-1\right) n}{c b}=\frac{\left(k_{1}-1\right) \ell n}{\left(k_{1} \ell+k_{0}\right) c}<1 .
$$

If $\ell \geq 2$, then $k_{1}(\ell-1)-2 \ell+1 \geq 3(\ell-1)-2 \ell+1=\ell-2 \geq 0$. This together with $\frac{n}{c}>2$ and $k_{1}-1 \geq k_{0}$ implies that $\frac{(c-b)\left(k_{1}-1\right) n}{c b} \geq \frac{2\left(k_{1}-1\right) \ell}{k_{1} \ell+k_{1}-1}=\frac{2 \ell k_{1}-2 \ell}{k_{1}(\ell+1)-1}=1+\frac{k_{1}(\ell-1)-2 \ell+1}{k_{1}(\ell+1)-1} \geq 1$, which is a contradiction to (3.6).

Finally, assume that $\ell=1$, so $a=2$. Therefore, the proposition follows from Lemma 2.7,

This completes the proof.

\subsection{Proof of Proposition 3.3}

In this subsection, we always assume that $\left\lceil\frac{n}{c}\right\rceil=\left\lceil\frac{n}{b}\right\rceil$, so $k_{1} \geq 2$, and we also assume that $k_{1} \leq$ $\frac{b}{a}$. Since $a \leq \frac{b}{k_{1}}$, by Lemma 2.4 we may assume that $\operatorname{gcd}\left(m_{1}, n\right)>1$ for every $m_{1} \in\left[\frac{k_{1} n}{c}, \frac{k_{1} n}{b}\right)$.

Since $s=\left\lfloor\frac{b}{a}\right\rfloor$, we have $2 \leq k_{1} \leq s \leq \frac{b}{a}<s+1$. By Lemma 2.5 we may assume that for every $t \in\left[0,\left\lfloor\frac{s}{2}\right\rfloor-1\right]$

$$
\left[\frac{(2 s-2 t-1) n}{2 b}, \frac{(s-t) n}{b}\right] \text { contains no integers co-prime to } n \text {. }
$$

We divide the proof of Proposition 3.3 into the following few lemmas.

Recall that by Remark 2.1 (1), we may always assume that $n>1000$. The next lemma provides an upper bound for $n$, which will be used frequently to obtain a contradiction by showing that $n \leq 1000$.

Lemma 3.7. If $u<\frac{n}{c}<\frac{n}{b}<v$ for some real numbers $u, v$ and $u\left(k_{1}-1\right)>s+1$, then

$$
n<\frac{u v\left(k_{1}-1\right)(s+1)}{u\left(k_{1}-1\right)-(s+1)} .
$$


Proof. Assume to the contrary that $n \geq \frac{u v\left(k_{1}-1\right)(s+1)}{u\left(k_{1}-1\right)-(s+1)}$. Then $b>\frac{n}{v} \geq \frac{u\left(k_{1}-1\right)(s+1)}{u\left(k_{1}-1\right)-(s+1)}$. Since $\frac{b}{a}<s+1, a>\frac{b}{s+1}$. Therefore,

$$
\begin{aligned}
& \frac{\left(k_{1}-1\right) n(a-1)}{b c}=\frac{k_{1}-1}{b} \times \frac{n}{c} \times(a-1)>\frac{k_{1}-1}{b} \times u \times\left(\frac{b}{s+1}-1\right) \\
= & \frac{u\left(k_{1}-1\right)}{s+1} \times \frac{b-(s+1)}{b} \geq \frac{u\left(k_{1}-1\right)}{s+1} \times \frac{\frac{u\left(k_{1}-1\right)(s+1)}{u\left(k_{1}-1\right)-(s+1)}-(s+1)}{\frac{u\left(k_{1}-1\right)(s+1)}{u\left(k_{1}-1\right)-(s+1)}} \\
= & 1,
\end{aligned}
$$

yielding a contradiction to (3.1).

Lemma 3.8. If the assumption is as in Proposition 3.3, then $k_{1} \leq 3$.

Proof. Assume to the contrary that $k_{1} \geq 4$. Recall that $k_{1} \leq s \leq 7$. We distinguish three cases according to the value of $k_{1}$.

Case 1. $6 \leq k_{1} \leq 7$. Then $6 \leq k_{1} \leq s \leq 7$. By Lemma 2.6 (iv) we have that $5 \mid n$ and $\frac{n}{b}<\frac{14}{5}$. Thus $2<\frac{n}{c}<\frac{n}{b}<\frac{14}{5}$. Applying Lemma 3.7 with $u=2$ and $v=\frac{14}{5}$, we infer that $n<112$, yielding a contradiction Remark 2.1 (1).

Case 2. $k_{1}=5$. Then $5=k_{1} \leq s \leq 7$. By Lemma 2.6 (ii) we have $\frac{n}{b}<4$.

If $3<\frac{n}{b}<4$, since $\left\lceil\frac{n}{c}\right\rceil=\left\lceil\frac{n}{b}\right\rceil$, we have $3<\frac{n}{c}<\frac{n}{b}<4$. Applying Lemma 3.7 with $u=3$ and $v=4$, we infer that $n<84$, yielding a contradiction again.

Now assume that $2<\frac{n}{b}<3$. Since $\left\lceil\frac{n}{c}\right\rceil=\left\lceil\frac{n}{b}\right\rceil$, we have $2<\frac{n}{c}<\frac{n}{b}<3$. If $s \leq 6$. Then applying Lemma 3.7 with $u=2$ and $v=3$, we infer that $n<168$, yielding a contradiction. If $s=7$, by Lemma [2.6 (ii) and (iv) we conclude that $\left[\frac{(2 s-2 t-1) n}{2 b}, \frac{(s-t) n}{b}\right]$ contains exactly one integer for each $t \in\left[0,\left\lfloor\frac{s}{2}\right\rfloor-1\right]$ and the integer in $\left[\frac{9 n}{2 b}, \frac{5 n}{b}\right]$ is divisible by 5 . Note that $9<\frac{9 n}{2 b}<\frac{27}{2}$ and $10<\frac{5 n}{b}<15$. We infer that $9<\frac{9 n}{2 b} \leq 10<\frac{5 n}{b}<11$. By the definition of $k_{1}$, $\left[\frac{5 n}{c}, \frac{5 n}{b}\right)$ contains an integer, so we conclude that $\frac{5 n}{c} \leq 10<\frac{5 n}{b}<11$. Thus $\frac{n}{c} \leq 2$, yielding a contradiction to that $c<\frac{n}{2}$.

Case 3. $k_{1}=4$. Then $4=k_{1} \leq s \leq 7$. By Lemma 2.6 (ii) we have $\frac{n}{b}<4$. We divide the proof into two subcases.

Subcase 3.1. $3<\frac{n}{b}<4$. Since $\left\lceil\frac{n}{c}\right\rceil=\left\lceil\frac{n}{b}\right\rceil$, we have $3<\frac{n}{c}<\frac{n}{b}<4$. Applying Lemma 3.7 with $u=3$ and $v=4$, we infer that $n<126$, a contradiction.

Subcase 3.2. $2<\frac{n}{b}<3$. Since $\left\lceil\frac{n}{c}\right\rceil=\left\lceil\frac{n}{b}\right\rceil$, we have $2<\frac{n}{c}<\frac{n}{b}<3$, so $8<\frac{4 n}{c} \leq m_{1}<\frac{4 n}{b}<12$. Since $\operatorname{gcd}(n, 6)>1$ and $\operatorname{gcd}\left(n, m_{1}\right)>1$, we infer that $m_{1} \in[10,11]$, so we have either $9<\frac{4 n}{c} \leq$ $10<\frac{4 n}{b}<12$ or $10<\frac{4 n}{c} \leq 11<\frac{4 n}{b}<12$.

First assume that $9<\frac{4 n}{c} \leq 10<\frac{4 n}{b}<12$. If $s \leq 5$, then applying Lemma 3.7 with $u=\frac{9}{4}$ and $v=3$, we infer that $n<162$, a contradiction. Now assume that $s=6$ or $s=7$. We shall show that $\frac{4 n}{b} \leq 11$. Assume to the contrary that $\frac{4 n}{b}>11$. Then $\frac{11}{4}<\frac{n}{b}<3$ and $[10,11] \subset\left[\frac{4 n}{c}, \frac{4 n}{b}\right)$. Since $\operatorname{gcd}\left(n, m_{1}\right)>1$ for every integer $m_{1} \in\left[\frac{4 n}{c}, \frac{4 n}{b}\right)$, we have $\operatorname{gcd}(n, 10)>1$ and $\operatorname{gcd}(n, 11)>1$. Since $n=p_{1}^{\alpha} \cdot p_{2}^{\beta}$, we infer that $n=5^{\alpha} \cdot 11^{\beta}$. Since $15<\frac{121}{8}<\frac{11 n}{2 b}<\frac{6 n}{b}<18$, we conclude that $\left[\frac{11 n}{2 b}, \frac{6 n}{b}\right]$ contains an integer which is co-prime to $n$, a contradiction to (3.7). Thus we must have $\frac{4 n}{b} \leq 11$. Then $\frac{5}{2}<\frac{n}{b} \leq \frac{11}{4}$, which implies that $11<\frac{45}{4}<\frac{9 n}{2 b}<\frac{5 n}{b}<\frac{55}{4}<14$. 
Suppose $x \in\left[\frac{9 n}{2 b}, \frac{5 n}{b}\right] \subset[12,13]$. By (3.7) we have $\operatorname{gcd}(n, x)>1$. Since $\operatorname{gcd}(n, 6)=1$, we infer that $x=13$ and hence $12<\frac{9 n}{2 b} \leq 13 \leq \frac{5 n}{b}<14$. Thus $\frac{8}{3}<\frac{n}{b} \leq \frac{11}{4}$, which implies that $\frac{11 n}{2 b} \leq \frac{121}{8}<16<\frac{6 n}{b}$. Therefore, $16 \in\left[\frac{11 n}{2 b}, \frac{6 n}{b}\right]$. By (3.7), we obtain that $\operatorname{gcd}(n, 16)>1$, a contradiction to $\operatorname{gcd}(n, 6)=1$.

Next assume that $10<\frac{4 n}{c} \leq 11<\frac{4 n}{b}<12$. If $s \leq 6$, then applying Lemma 3.7 with $u=\frac{5}{2}$ and $v=3$, we infer that $n<315$. Now assume that $s=7$. Note that $\frac{11}{4} \leq \frac{n}{b}<3$. Then $12<\frac{99}{8} \leq \frac{9 n}{2 b}<\frac{5 n}{b}<15$ and thus $\left[\frac{9 n}{2 b}, \frac{5 n}{b}\right] \subset[13,14]$. However, by Lemma 2.6 (iv), we obtain that $\left[\frac{9 n}{2 b}, \frac{5 n}{b}\right]$ contains an integer which is divisible by 5 , yielding a contradiction.

Lemma 3.9. If $k_{1}=3$, then $\operatorname{ind}(S)=1$.

Proof. We remark that since $k_{1}=3$, we conclude that $\left[\frac{2 n}{c}, \frac{2 n}{b}\right)$ contains no integers and $\left[\frac{k_{2} n}{c}, \frac{k_{2} n}{b}\right)$ contains at least one integer where $k_{2} \geq 3$.

Note that $3=k_{1} \leq s \leq 7$. By Lemma 2.6 (i) we have $\frac{n}{b}<6$. We distinguish three cases according to the value of $\frac{n}{b}$.

Case 1. $4<\frac{n}{b}<6$. By Lemma $\left[2.6\right.$ (ii) we must have $s=3$. Since $\left\lceil\frac{n}{c}\right\rceil=\left\lceil\frac{n}{b}\right\rceil$, we infer that $4<\frac{n}{c}<\frac{n}{b}<6$. Applying Lemma 3.7 with $u=4$ and $v=6$, we infer that $n<48$, a contradiction to Remark 2.1 (1).

Case 2. $3<\frac{n}{b}<4$. By Lemma 2.6 (iv), we must have $s \leq 5$.

Since $\left\lceil\frac{n}{c}\right\rceil=\left\lceil\frac{n}{b}\right\rceil$, we have $3<\frac{n}{c}<\frac{n}{b}<4$ and thus $9<\frac{3 n}{c} \leq m_{1}<\frac{3 n}{b}<12$. We infer that $m_{1} \in[10,11]$, so we have either $9<\frac{3 n}{c} \leq 10<\frac{3 n}{b}<12$ or $10<\frac{3 n}{c} \leq 11<\frac{3 n}{b}<12$. If $s \leq 4$, then applying Lemma 3.7 with $u=3$ and $v=4$, we infer that $n<120$, a contradiction. Next assume that $s=5$.

Suppose that $9<\frac{3 n}{c} \leq 10<\frac{3 n}{b}<12$. We will show that $\frac{3 n}{b}<11$. Assume to the contrary that $\frac{3 n}{b} \geq 11$, then $\frac{2 n}{c} \leq \frac{20}{3}<7<\frac{22}{3}<\frac{2 n}{b}$, which is impossible since $\left[\frac{2 n}{c}, \frac{2 n}{b}\right)$ contains no integers. Therefore, we have that $\frac{3 n}{b}<11$, and then $\frac{10}{3}<\frac{n}{b}<\frac{11}{3}$. Hence $15<\frac{9 n}{2 b}<\frac{5 n}{b}<$ $\frac{55}{3}<19$ and $11<\frac{35}{3}<\frac{7 n}{2 b}<\frac{4 n}{b}<\frac{44}{3}<15$. Thus $\left[\frac{9 n}{2 b}, \frac{5 n}{b}\right] \subset[16,18]$ and $\left[\frac{7 n}{2 b}, \frac{4 n}{b}\right] \subset[12,14]$. Suppose $x \in\left[\frac{9 n}{2 b}, \frac{5 n}{b}\right]$ and $y \in\left[\frac{7 n}{2 b}, \frac{4 n}{b}\right]$. Then $5 \nmid x y$. Since $n=p_{1}^{\alpha} \cdot p_{2}^{\beta}$, by Lemma 2.6 (iii) we may assume that $p_{1} \mid x$ and $p_{2} \mid y$. Hence $p_{1} p_{2} \mid x y$. Note that $m_{1}=10$. Since $\operatorname{gcd}\left(n, m_{1}\right)>1$ and $\operatorname{gcd}(n, 6)=1$, we have $5 \mid n$. Hence $5 \in\left\{p_{1}, p_{2}\right\}$ and thus $5 \mid x y$, yielding a contradiction.

Suppose that $10<\frac{3 n}{c} \leq 11<\frac{3 n}{b}<12$. Then applying Lemma 3.7 with $u=\frac{10}{3}$ and $v=4$, we infer that $n<240$, a contradiction.

Case 3. $2<\frac{n}{b}<3$. Since $\left\lceil\frac{n}{c}\right\rceil=\left\lceil\frac{n}{b}\right\rceil$, we have $2<\frac{n}{c}<\frac{n}{b}<3$, and thus $6<\frac{3 n}{c} \leq m_{1}<\frac{3 n}{b}<9$. Since $\operatorname{gcd}(n, 6)>1$ and $\operatorname{gcd}\left(n, m_{1}\right)>1$, we infer that $m_{1}=7$ and thus $6<\frac{3 n}{c} \leq 7<\frac{3 n}{b}<8$. Therefore, $7 \mid n$ and $\frac{7}{3}<\frac{n}{b}<\frac{8}{3}$.

First assume that $s \geq 5$. Note that $\frac{9 n}{2 b}<12$. We will show that $\frac{5 n}{b}<12$. If $\frac{5 n}{b} \geq 12$, then $12 \in\left[\frac{9 n}{2 b}, \frac{5 n}{b}\right]$. By (3.7), we have $\operatorname{gcd}(n, 12)>1$, a contradiction to that $\operatorname{gcd}(n, 6)=1$. Therefore $\frac{7}{3}<\frac{n}{b}<\frac{12}{5}$.

If $s=7$, by Lemma 2.6 (iv), we have that the integer in $\left[\frac{13 n}{2 b}, \frac{7 n}{b}\right]$ is divisible by 5 , which is impossible because $15<\frac{13 n}{2 b}<\frac{7 n}{b}<17$.

If $5 \leq s \leq 6$, we have that $8<\frac{49}{6}<\frac{7 n}{2 b}<\frac{4 n}{b}<\frac{48}{5}<10$. Hence $9 \in\left[\frac{7 n}{2 b}, \frac{4 n}{b}\right]$. By (3.7), we have $\operatorname{gcd}(n, 9)>1$, a contradiction again. 
Next assume that $s=4$. We have that $8<\frac{49}{6}<\frac{7 n}{2 b}<\frac{4 n}{b}<\frac{32}{3}<11$ and thus $\left[\frac{7 n}{2 b}, \frac{4 n}{b}\right] \subset[9,10]$. By (3.7) and $\operatorname{gcd}(n, 6)=1$, we have $\left[\frac{7 n}{2 b}, \frac{4 n}{b}\right]=\{10\}$ and thus $9<\frac{7 n}{2 b} \leq 10 \leq \frac{4 n}{b}<11$, so $\frac{18}{7}<\frac{n}{b}<\frac{8}{3}$. If $\frac{4 n}{c} \leq 10$, then $\frac{2 n}{c} \leq 5<\frac{2 n}{b}$, which is impossible since $\left[\frac{2 n}{c}, \frac{2 n}{b}\right)$ contains no integers. So we must have that $10<\frac{4 n}{c}<\frac{4 n}{b}<11$, yielding a contradiction to the assumption that $\left[\frac{4 n}{c}, \frac{4 n}{b}\right)$ contains an integer.

Finally, assume that $s=3$. If $\frac{9}{4}<\frac{n}{c}<\frac{n}{b}<3$, then applying Lemma 3.7 with $u=\frac{9}{4}$ and $v=3$, we infer that $n<108$, a contradiction. Thus $\frac{n}{c} \leq \frac{9}{4}$, and then $\frac{2 n}{c} \leq \frac{9}{2}<5$. Since $\left[\frac{2 n}{c}, \frac{2 n}{b}\right)$ contains no integers, we have $\frac{2 n}{c}<\frac{2 n}{b} \leq 5$. Since $\frac{n}{b}>\frac{7}{3}>\frac{9}{4}$, we have that $\frac{4 n}{c} \leq 9<\frac{4 n}{b}$. Let $m=9$ and $k=4$. Since $\frac{2 n}{5}<b<c<\frac{n}{2}, m a=9 \times(c-b+1) \leq 9 \times\left(\frac{n-1}{2}-\frac{2 n}{5}+1\right)<n$, so the lemma follows from Lemma 2.3 (1).

Lemma 3.10. Let $k_{1}=2,4<\frac{2 n}{c} \leq 5<\frac{2 n}{b}<6$ and $a \leq \frac{b}{2}$. Then $\operatorname{ind}(S)=1$.

Proof. Note that $m_{1}=5$ and $b \geq 2 a \geq 6$. Since $\operatorname{gcd}\left(n, m_{1}\right)>1$ we have $5 \mid n$. Since $4<\frac{2 n}{c} \leq 5<\frac{2 n}{b}<6$, we obtain that $\frac{n}{3} \leq b<\frac{2 n}{5} \leq c<\frac{n}{2}$.

Recall that $n>1000$. We shall show that either there exist positive integers $k, m$ satisfying the condition of Lemma 2.3 (1), or there exists a positive integer $M$ satisfying the condition of Lemma 2.3 (2), and then the lemma follows from Lemma 2.3 .

By the definition of $k_{1}$, we conclude that $\left[\frac{k_{2} n}{c}, \frac{k_{2} n}{b}\right)$ contains at least one integer for each $k_{2} \geq k_{1}=2$. Note that $6<\frac{3 n}{c}<\frac{3 n}{b}<9$. We distinguish three cases.

Case 1. $7<\frac{3 n}{c} \leq 8<\frac{3 n}{b}<9$. Then $\frac{n}{3}<b<\frac{3 n}{8} \leq c<\frac{3 n}{7}$.

Note that $\operatorname{gcd}(n, 8)=1$. Let $m=8$ and $k=3(\leq b=6)$. Since $\frac{n}{3}<b<c<\frac{3 n}{7}$, $m a=m(c-b+1) \leq 8 \times\left(\frac{3 n-1}{7}-\frac{n+1}{3}+1\right)<n$, and we are done.

Case 2. $6<\frac{3 n}{c} \leq 7<\frac{3 n}{b}<8$. Then $\frac{3 n}{8}<b<\frac{2 n}{5}<\frac{3 n}{7} \leq c<\frac{n}{2}$.

If $\operatorname{gcd}(7, n)=1$, then let $m=7$ and $k=3$. Since $\frac{3 n}{8}<b<c<\frac{n}{2}, m a=m(c-b+1) \leq$ $7 \times\left(\frac{n-1}{2}-\frac{3 n+1}{8}+1\right)<n$, and we are done.

Next assume that $7 \mid n$, i.e. $n=5^{\alpha} \cdot 7^{\beta}$. Note that $8<\frac{4 n}{c} \leq 10<\frac{4 n}{b}<12$.

If $9 \notin\left[\frac{4 n}{c}, \frac{4 n}{b}\right)$, then $\frac{4 n}{c}>9$. Let $m=12$ and $k=5$. Since $\frac{5 n}{c}<12<\frac{5 n}{b}$ and $\frac{3 n}{8}<b<c<\frac{4 n}{9}$, we have $m a=m(c-b+1) \leq 12 \times\left(\frac{4 n-1}{9}-\frac{3 n+1}{8}+1\right)<n$, and we are done.

If $9 \in\left[\frac{4 n}{c}, \frac{4 n}{b}\right)$, then $\frac{4 n}{c} \leq 9<10<\frac{4 n}{b}$ and thus $\frac{3 n}{8}<b<\frac{2 n}{5}<\frac{4 n}{9}<c<\frac{n}{2}$. So

$$
8 n+\frac{n}{2}<\frac{69 n}{8}<23 b<\frac{46 n}{5}<9 n+\frac{n}{2}<10 n<\frac{92 n}{9}<23 c<\frac{23 n}{2}=11 n+\frac{n}{2} .
$$

Note that $a=c-b+1 \leq \frac{n-1}{2}-\frac{3 n+1}{8}+1=\frac{n+3}{8}$. If $a>\frac{n}{8}$, then let $M=12$ (note that $\operatorname{gcd}(n, 12)=1)$. We obtain that $|M a|_{n}>\frac{n}{2}$ and $|M b|_{n}>\frac{n}{2}$, and we are done. If $a<\frac{n}{8}$, since $\operatorname{gcd}(9, n)=1$, we may assume that $a>\frac{n}{9}$ (for otherwise, let $m=9$ and $k=4$, we have $m a<n$, and we are done). Then $\frac{n}{9}<a<\frac{n}{8}$, and thus

$$
2 n+\frac{n}{2}<\frac{23 n}{9}<23 a<\frac{23 n}{8}<3 n .
$$

If $23 c<11 n$, then $\frac{n}{9}<a=c-b+1 \leq \frac{11 n-1}{23}-\frac{3 n+1}{8}+1=\frac{19 n+57}{184}$, which implies that $n<40$, yielding a contradiction. So we must have $23 c>11 n$. Similarly, we can show that $23 b<9 n$. Then $|23|_{n}+|23 c|_{n}+|23(n-b)|_{n}+|23(n-a)|_{n}=23+(23 c-11 n)+(9 n-23 b)+(3 n-23 a)=n$ and we are done.

Case 3. $6<\frac{3 n}{c} \leq 7<8<\frac{3 n}{b}<9$. Then $\frac{n}{3}<b<\frac{3 n}{8}<\frac{3 n}{7} \leq c<\frac{n}{2}$. 
Note that $a=c-b+1 \leq \frac{n-1}{2}-\frac{n+1}{3}+1=\frac{n+1}{6}$ and $\operatorname{gcd}(n, 3)=1$. If $a>\frac{n}{6}$, let $M=3$. Then $|3 a|_{n}>\frac{n}{2}$ and $|3 c|_{n}<\frac{n}{2}$, and we are done. Next assume that $a<\frac{n}{6}$.

Subcase 3.1. $\operatorname{gcd}(7, n)=\operatorname{gcd}(11, n)=1$.

We may assume that $a>\frac{n}{7}$ (for otherwise, if let $m=7$ and $k=3$, we have $m a<n$, so the lemma follows from Lemma $2.3(1))$. Hence $n<11 a<2 n$. Also, we have that $3 n<\frac{11 n}{3}<$ $11 b<\frac{33 n}{8}<5 n$ and $4 n<\frac{33 n}{7}<11 c<\frac{11 n}{2}<6 n$.

If $11 b<4 n$ and $11 c>5 n$, we have $|11|_{n}+|11 c|_{n}+|11(n-b)|_{n}+|11(n-a)|_{n}=11+11 c-$ $5 n+4 n-11 b+2 n-11 a=n$ and thus $\operatorname{ind}(S)=1$.

If $11 b>4 n$ and $11 c<5 n$, we have $|11|_{n}+|11 c|_{n}+|11(n-b)|_{n}+|11(n-a)|_{n}=11+11 c-$ $4 n+5 n-11 b+2 n-11 a=3 n$ and thus $\operatorname{ind}(S)=1$ (by Remark 2.1 (2)).

If $11 b<4 n$ and $11 c<5 n$, then $\frac{n}{7}<a=c-b+1 \leq \frac{5 n-1}{11}-\frac{n+1}{3}+1=\frac{4 n+19}{33}$, so $n<27$, a contradiction.

If $11 b>4 n$ and $11 c>5 n$, then $\frac{n}{7}<a=c-b+1 \leq \frac{n-1}{2}-\frac{4 n+1}{11}+1=\frac{3 n+9}{22}$, so $n<63$, again a contradiction.

Subcase 3.2. $n=5^{\alpha} \cdot 11^{\beta}$.

As in Subcase 3.1, we may assume that $a>\frac{n}{7}$. Then $\frac{3 n}{2}<\frac{13 n}{7}<13 a<\frac{13 n}{6}<\frac{5 n}{2}<4 n<$ $\frac{13 n}{3}<13 b<\frac{39 n}{8}<5 n<\frac{11 n}{2}<\frac{39 n}{7}<13 c<\frac{13 n}{2}$.

If $13 c<6 n$, then $\frac{n}{7}<a=c-b+1 \leq \frac{6 n-1}{13}-\frac{n+1}{3}+1=\frac{5 n+23}{39}$, so $n<41$, yielding a contradiction. Hence we must have that $13 c>6 n$, and then $|13 c|_{n}<\frac{n}{2}$. If $13 a<2 n$ or $13 b>\frac{9 n}{2}$, then $|13 a|_{n}>\frac{n}{2}$ or $|13 b|_{n}>\frac{n}{2}$. Since $\operatorname{gcd}(n, 13)=1$, the lemma follows from Lemma 2.3 (2) with $M=13$.

Next assume that $13 a>2 n$ and $13 b<\frac{9 n}{2}$. Then $\frac{2 n}{13}<a<\frac{n}{6}$ and $\frac{n}{3}<b<\frac{9 n}{26}$. Therefore,

$$
\frac{5 n}{2}<\frac{34 n}{13}<17 a<\frac{17 n}{6}<3 n<\frac{11 n}{2}<\frac{17 n}{3}<17 b<\frac{153 n}{26}<6 n .
$$

We infer that $|17 a|_{n}>\frac{n}{2}$ and $|17 b|_{n}>\frac{n}{2}$. Since $\operatorname{gcd}(n, 17)=1$, the lemma follows from Lemma 2.3 (2) with $M=17$.

Subcase 3.3. $n=5^{\alpha} \cdot 7^{\beta}$.

As in Subcase 3.1, we may assume that $a>\frac{n}{8}$. By using a similar argument in Subcase 3.2, we can complete the proof with $M=11$ or $M=13$.

Lemma 3.11. If $k_{1}=2$, then $\operatorname{ind}(S)=1$.

Proof. Note that $2=k_{1} \leq s \leq 7$. By Lemma 2.6 (i) we have $\frac{n}{b}<6$. We distinguish several cases according to the value of $\frac{n}{b}$.

Case 1. $5<\frac{n}{b}<6$. Since $\left\lceil\frac{n}{c}\right\rceil=\left\lceil\frac{n}{b}\right\rceil$, we have that $5<\frac{n}{c}<\frac{n}{b}<6$. If $s \geq 4$, by Lemma 2.6 (ii) we have $\frac{n}{b}<4$, which yields a contradiction. If $2 \leq s \leq 3$, applying Lemma 3.7 with $u=5$ and $v=6$, we infer that $n<120$, yielding a contradiction again.

Case 2. $4<\frac{n}{b} \leq 5$. Since $\left\lceil\frac{n}{c}\right\rceil=\left\lceil\frac{n}{b}\right\rceil$, we infer that $4<\frac{n}{c}<\frac{n}{b} \leq 5$, and then $8<\frac{2 n}{c} \leq$ $m_{1}<\frac{2 n}{b} \leq 10$, so $m_{1}=9$. Now $\operatorname{gcd}\left(n, m_{1}\right)>1$, i.e, $\operatorname{gcd}(9, n)>1$ yields a contradiction to $\operatorname{gcd}(n, 6)=1$.

Case 3. $3<\frac{n}{b}<4$. Since $\left\lceil\frac{n}{c}\right\rceil=\left\lceil\frac{n}{b}\right\rceil$, we have $3<\frac{n}{c}<\frac{n}{b}<4$, thus $6<\frac{2 n}{c} \leq m_{1}<\frac{2 n}{b}<8$. Hence $m_{1}=7$ and thus $\frac{7}{2}<\frac{n}{b}<4$. Since $\operatorname{gcd}\left(n, m_{1}\right)>1$, we obtain that $7 \mid n$.

If $s \geq 6$, by Lemma 2.6 (iv) we have that $\frac{n}{b}<\frac{14}{5}<3$, a contradiction. 
If $4 \leq s \leq 5$, by Lemma 2.6 (ii), we can assume that $\left[\frac{(2 s-2 t-1) n}{2 b}, \frac{(s-t) n}{b}\right]$ contains only one integer for each $t \in\left[0,\left\lfloor\frac{s}{2}\right\rfloor-1\right]$. Since $\frac{7}{2}<\frac{n}{b}<4,12<\frac{49}{4}<\frac{7 n}{2 b}<14<\frac{4 n}{b}<16$. Hence 14 is the only integer in $\left[\frac{7 n}{2 b}, \frac{4 n}{b}\right]$, and thus $13<\frac{7 n}{2 b}<14<\frac{4 n}{b}<15$. Then $\frac{26}{7}<\frac{n}{b}<\frac{15}{4}$. If $s=5$, then $16<\frac{117}{7}<\frac{9 n}{2 b}<\frac{135}{8}<17<18<\frac{130}{7}<\frac{5 n}{b}<\frac{75}{4}<19$, a contradiction (since $\left[\frac{9 n}{2 b}, \frac{5 n}{b}\right]$ contains only one integer). If $s=4$, then $9<\frac{65}{7}<\frac{5 n}{2 b}<\frac{75}{8}<10<11<\frac{78}{7}<\frac{3 n}{b}<\frac{45}{4}<12$, a contradiction (since $\left[\frac{5 n}{2 b}, \frac{3 n}{b}\right]$ contains only one integer).

Next assume that $s=3$. Since $\frac{7}{2}<\frac{n}{b}<4,8<\frac{35}{4}<\frac{5 n}{2 b}<10<\frac{21}{2}<\frac{3 n}{b}<12$ and thus $10 \in\left[\frac{5 n}{2 b}, \frac{3 n}{b}\right]$. By (3.7) we have $\operatorname{gcd}(10, n)>1$. Since $\operatorname{gcd}(n, 6)=1$, we infer that $5 \mid n$ and $n=5^{\alpha} \cdot 7^{\beta}$. Then $\operatorname{gcd}(11, n)=\operatorname{gcd}(9, n)=1$. So by (3.7), both 9 and 11 are not in $\left[\frac{5 n}{2 b}, \frac{3 n}{b}\right]$. Therefore $9<\frac{5 n}{2 b}<10<\frac{3 n}{b}<11$ and thus $\frac{18}{5}<\frac{n}{b}<\frac{11}{3}$. Note that $12<\frac{4 n}{c} \leq 14<\frac{4 n}{b}<16$ and $\operatorname{gcd}(n, 13)=1$. If $13 \in\left[\frac{4 n}{c}, \frac{4 n}{b}\right)$, let $m=13$ and $k=4$. Since $3<\frac{n}{c}<\frac{n}{b}<\frac{11}{3}$, we have $\frac{3 n}{11}<b<c<\frac{n}{3}$. Then $m a=13 \times(c-b+1) \leq 13 \times\left(\frac{n-1}{3}-\frac{3 n+1}{11}+1\right)<n$ and we are done. Hence we may assume that $13 \notin\left[\frac{4 n}{c}, \frac{4 n}{b}\right)$. Then $\frac{4 n}{c}>13$ and thus $c<\frac{4 n}{13}$. Since $\frac{n}{c}<\frac{10}{3}<\frac{18}{5}<\frac{n}{b}$, we obtain that $\frac{5 n}{c}<18<\frac{5 n}{b}$. Let $m=18$ and $k=5$. Since $\operatorname{gcd}(18, n)=1$ and $\frac{3 n}{11}<b<c<\frac{4 n}{13}$, $m a=18 \times(c-b+1) \leq 18 \times\left(\frac{4 n-1}{13}-\frac{3 n+1}{11}+1\right)<n$, and we are done.

Finally, assume that $s=2$. Since $\frac{3 n}{2 b}<\frac{3}{2} \times 4=6<7<\frac{2 n}{b}$, we have $6 \in\left[\frac{3 n}{2 b}, \frac{2 n}{b}\right]$. By (3.7) we have $\operatorname{gcd}(6, n)>1$, a contradiction.

Case 4. $2<\frac{n}{b}<3$. Since $\left\lceil\frac{n}{c}\right\rceil=\left\lceil\frac{n}{b}\right\rceil$, we have $2<\frac{n}{c}<\frac{n}{b}<3$, and thus $4<\frac{2 n}{c} \leq m_{1}<\frac{2 n}{b}<6$, so $m_{1}=5$. Since $\operatorname{gcd}\left(n, m_{1}\right)>1$, we have $5 \mid n$. The result now follows from Lemma 3.10

Now Proposition 3.3 follows immediately from Lemmas 3.8 , 3.9 and 3.11 .

\section{Applications}

In this section, we give two applications of our main result. It is shown that our main result (Theorem 1.3) implies that Problem 1.2 has an affirmative answer for the case when the order of $G$ is a prime power as well as the case when $|G|$ is a product of two different primes. We first remark that by using a similar, but much simpler and shorter, argument as in the proof of Proposition 2.2, we obtain the following result.

Proposition 4.1. [10, Proposition 2.1] Let $|G|=n=p^{\alpha}$ where $p \in \mathbb{P}$ and $\operatorname{gcd}(p, 6)=1, \alpha \in \mathbb{N}$. Let $S=(g)(c g)((n-b) g)((n-a) g)$ be a minimal zero-sum sequence over $G$ such that $\operatorname{ord}(g)=n$, $1+c=a+b$ and $1<a \leq b<c<\frac{n}{2}$. Then $\operatorname{ind}(S)=1$.

When the order of the group $G$ is a prime power (i.e. $|G|=p^{k}$ ), without loss of generality, we may assume that each minimal zero-sum sequence $\mathrm{S}$ can be written in the following form:

$$
S=\left(p^{l} g\right)\left(\left(p^{l} x_{1}\right) g\right)\left(\left(p^{l} x_{2}\right) g\right)\left(\left(p^{l} x_{3}\right) g\right), \text { where } 1 \leq x_{1}, x_{2}, x_{3}<\frac{n}{p^{l}}=p^{k-l} \text { and }|G|=\operatorname{ord}(g) .
$$

Let $g_{1}=p^{l} g$, and then $S$ can be rewritten as $T=\left(g_{1}\right)\left(x_{1} g_{1}\right)\left(x_{2} g_{1}\right)\left(x_{3} g_{1}\right)$, which can regarded as a minimal zero-sum sequence over the subgroup $G_{1}=\left\langle g_{1}\right\rangle$. The question of determining whether or not the index of $S$ (over $G$ ) is 1 is reduced to that of determining whether or not the index of $T$ (over $G_{1}$ ) is 1. By applying Proposition 4.1 (and some simple observations), it is easy to show the latter is always the case, answering Problem 1.2 affirmatively for the prime power case. 
Theorem 4.2 (Li, Plyley, Yuan and Zeng (2010)). Let $G$ be a cyclic group of prime power order such that $\operatorname{gcd}(|G|, 6)=1$. Then every minimal zero-sum sequence $S$ over $G$ of length $|S|=4$ has $\operatorname{ind}(S)=1$.

We note that when the order of $G$ is not necessarily a prime power (say, for example, $|G|=$ $p^{\alpha} q^{\beta}$ is a product of two prime powers), the above mentioned reduction is not always possible as shown in the following example.

Example 4.3. Let $|G|=n=\left(5^{2}\right)(7)=175$ and $S=(5 g)(135 g)(77 g)(133 g)$ where $\operatorname{ord}(g)=n$. Clearly, $S$ cannot be reduced to the simplified form as described in (4.1). Note that $|4 \times 5|_{n}+$ $|4 \times 135|_{n}+|4 \times 77|_{n}+|4 \times 133|_{n}=20+15+133+7=175=n$, so we obtain that $\operatorname{ind}(S)=1$.

The obstruction mentioned in the above example prevents us to apply directly our main result to answer the problem affirmatively for the general case when the order of $G$ is a product of two prime powers. However, our main result does apply to several new special situations. We conclude the paper by providing one such case.

Theorem 4.4. If $|G|=p q$ with $p \neq q$ primes and $\operatorname{gcd}(p q, 6)=1$, then Problem 1.2 has an affirmative answer.

Proof. Let $S=\left(x_{1} g\right)\left(x_{2} g\right)\left(x_{3} g\right)\left(x_{4} g\right)$ be a minimal zero-sum sequence over $G$ such that ord $(g)=$ $n$. By Theorem [1.3, we may assume that $\operatorname{gcd}\left(n, x_{i}\right)>1$ for every $i \in[1,4]$.

If $\operatorname{gcd}\left(n, x_{i}\right)=p$ for every $i \in[1,4]$, let $g_{1}=p g$. As mentioned above $S$ can be rewritten as $T=\left(\frac{x_{1}}{p} g_{1}\right)\left(\frac{x_{2}}{p} g_{1}\right)\left(\frac{x_{3}}{p} g_{1}\right)\left(\frac{x_{4}}{p} g_{1}\right)$, which can be regarded as a minimal zero-sum sequence over the subgroup $G_{1}=\left\langle g_{1}\right\rangle$, where $\left|G_{1}\right|=\operatorname{ord}\left(g_{1}\right)=q$. By Theorem 4.2, we have $\operatorname{ind}(T)=1$ in $G_{1}$, i.e. there exists $m \in[1, q-1]$ such that $\operatorname{gcd}(m, q)=1$ and $\left|\frac{m x_{1}}{p}\right|_{q}+\cdots+\left|\frac{m x_{4}}{p}\right|_{q}=q$. Then $\left|m x_{1}\right|_{p q}+\cdots+\left|m x_{4}\right|_{p q}=\left|(m+q) x_{1}\right|_{p q}+\cdots+\left|(m+q) x_{4}\right|_{p q}=p q(*)$. Note that either $\operatorname{gcd}(m, p q)=1$ or $\operatorname{gcd}(m+q, p q)=1$. Now $(*)$ implies that $\operatorname{ind}(S)=1$.

If $\operatorname{gcd}\left(n, x_{i}\right)=q$ for every $i \in[1,4]$, let $g_{2}=q g$. As above we obtain that $\operatorname{ind}(S)=1$.

Next without loss of generality, we may assume that $\operatorname{gcd}\left(n, x_{1}\right)=\operatorname{gcd}\left(n, x_{2}\right)=p$ and $\operatorname{gcd}\left(n, x_{3}\right)=\operatorname{gcd}\left(n, x_{4}\right)=q$. Since $x_{1}+x_{2}+x_{3}+x_{4} \equiv 0(\bmod p q)$, we must have $q \mid x_{1}+x_{2}$. Then $p q \mid x_{1}+x_{2}$, yielding a contradiction to the assumption that $S$ is minimal zero-sum sequence. This completes the proof.

\section{REFERENCES}

[1] S.T. Chapman, M. Freeze, and W.W Smith, Minimal zero sequences and the strong Davenport constant, Discrete Math. 203(1999), 271-277.

[2] S.T. Chapman, and W.W Smith, A characterization of minimal zero-sequences of index one in finite cyclic groups, Integers 5(1)(2005), Paper A27, 5p.

[3] W. Gao, Zero sums in finite cyclic groups, Integers 0 (2000), Paper A14, 9p.

[4] W. Gao and A. Geroldinger, On products of $k$ atoms, Monatsh. Math. 156 (2009), 141-157.

[5] W. Gao, Y. Li, J. Peng, P. Plyley and G. Wang On the index of sequences over cyclic groups (English), Acta Arith. 148, No. 2, (2011) 119-134.

[6] A. Geroldinger, On non-unique factorizations into irreducible elements. II, Number Theory, Vol II Budapest 1987, Colloquia Mathematica Societatis Janos Bolyai, vol. 51, North Holland, 1990, 723-757.

[7] A. Geroldinger, Additive group theory and non-unique factorizations, Combinatorial Number Theory and Additive Group Theory (A. Geroldinger and I. Ruzsa, eds.), Advanced Courses in Mathematics CRM Barcelona, Birkhäuser, 2009, pp. $1-86$. 
MINIMAL ZERO-SUM SEQUENCES OF LENGTH FOUR

[8] A. Geroldinger and F. Halter-Koch, Non-Unique Factorizations. Algebraic, Combinatorial and Analytic Theory, Pure and Applied Mathematics, Vol. 278, Chapman \& Hall/CRC, 2006.

[9] D. Kleitman and P. Lemke, An addition theorem on the integers modulo n, J. Number Theory 31(1989), 335-345.

[10] Y. Li, C. Plyley, P. Yuan and X. Zeng, Minimal zero sum sequences of length four over finite cyclic groups, Journal of Number Theory. 130 (2010), 2033 - 2048.

[11] V. Ponomarenko, Minimal zero sequences of finite cyclic groups, Integers 4(2004), Paper A24, 6p.

[12] S. Savchev and F. Chen, Long zero-free sequences in finite cyclic groups, Discrete Math. 307(2007), 2671-2679.

[13] X. Xia and P. Yuan, Indexes of insplitable minimal zero-sum sequences of length $l\left(C_{n}\right)-1$, Discrete Math. 310(2010)1127-1133.

[14] P. Yuan, On the index of minimal zero-sum sequences over finite cyclic groups, J. Combin. Theory Ser. A 114(2007), 1545-1551.

Department of Mathematics, Brock University, St. Catharines, Ontario, Canada L2S 3A1

E-mail address: yli@brocku.ca

College of Science, Civil Aviation University of China, Tianjin 300300, P.R. China

E-mail address: jtpeng1982@yahoo.com.cn 